\title{
POSET IDEALS OF P-PARTITIONS AND GENERALIZED LETTERPLACE AND DETERMINANTAL IDEALS
}

\author{
GUNNAR FLØYSTAD
}

\begin{abstract}
For any finite poset $P$ we have the poset of isotone maps $\operatorname{Hom}(P, \mathbb{N})$, also called $P^{\text {op }}$-partitions. To any poset ideal $\mathcal{J}$ in $\operatorname{Hom}(P, \mathbb{N})$, finite or infinite, we associate monomial ideals: the letterplace ideal $L(\mathcal{J}, P)$ and the Alexander dual coletterplace ideal $L(P, \mathcal{J})$, and study them. We derive a class of monomial ideals in $\mathbb{k}\left[x_{p}, p \in P\right]$ called $P$-stable. When $P$ is a chain we establish a duality on strongly stable ideals. We study the case when $\mathcal{J}$ is a principal poset ideal. When $P$ is a chain we construct a new class of determinantal ideals which generalizes ideals of maximal minors and whose initial ideals are letterplace ideals of principal poset ideals.
\end{abstract}

\section{INTRODUCTION}

For a finite poset $P$ the isotone maps $P \rightarrow \mathbb{N}$ form a poset $\operatorname{Hom}(P, \mathbb{N})$ where two maps $\phi \leq \psi$ if $\phi(p) \leq \psi(p)$ for every $p \in P$. Denoting by $P^{\text {op }}$ the opposite poset, $\operatorname{Hom}(P, \mathbb{N})$ identifies with $P^{o p}$-partitions, originally introduced and studied by R.Stanley in the classic [12], see also [13, Section 4.5]. (For a poset $Q$ a $Q$-partition is a map $Q \rightarrow \mathbb{N}$ such that $q_{1} \leq q_{2}$ implies $\phi\left(q_{1}\right) \geq \phi\left(q_{2}\right)$.) In [6] the author together with B.Greve and J.Herzog introduced letterplace and co-letterplace ideals, monomial ideals in a polynomial ring, associated to poset ideals in $\operatorname{Hom}(P,[n])$ where $[n]=\{1<2<\cdots<$ $n\}$ is the chain. These identify naturally as the finite poset ideals in $\operatorname{Hom}(P, \mathbb{N})$. Here we generalize this by associating letterplace and co-letterplace ideals to any poset ideal $\mathcal{J}$ of $\operatorname{Hom}(P, \mathbb{N})$ or, alternatively formulated, to any poset ideal in the poset of $P^{\text {op }}$ partitions.

Letterplace and co-letterplace ideals. To any isotone map $\phi: P \rightarrow \mathbb{N}$ there is associated two natural subsets of $P \times \mathbb{N}$. The first is the graph $\Gamma \phi$, the second we call the ascent $\Lambda \phi$ and is $\{(p, i) \mid \phi(q) \leq i<\phi(p)$ for every $q<p\}$. Denote by $\mathbb{k}\left[x_{P \times \mathbb{N}}\right]$ the polynomial ring in the infinite number of variables $x_{p, i}$ where $p \in P$ and $i \in \mathbb{N}$. The co-letterplace ideal associated to a finite poset ideal $\mathcal{J} \subseteq \operatorname{Hom}(P, \mathbb{N})$ is a monomial ideal $L(P, \mathcal{J})$ in $\mathbb{k}\left[x_{P \times \mathbb{N}}\right]$ whose generators are given by monomials associated to graphs $\Gamma \phi \subseteq P \times \mathbb{N}$ for $\phi$ in $\mathcal{J}$. For a general poset ideal $\mathcal{J}$ the generators correspond to graphs of certain isotone maps $\phi: I \rightarrow \mathbb{N}$ where the $I$ are poset ideals in $P$. The letterplace ideal $L(\mathcal{J}, P)$ in $\mathbb{k}\left[x_{P \times \mathbb{N}}\right]$ has generators given by the ascents $\Lambda \phi$ of $\phi$ 's in the complement filter $\mathcal{J}^{c}$ of $\operatorname{Hom}(P, \mathbb{N})$. These monomial ideals are Alexander dual, Proposition 1.11. Furthermore these ideals have finite minimal generating sets, so if we let $S$ be the set of all variables occurring in these generators, the support of these

Date: April 26, 2018.

2010 Mathematics Subject Classification. Primary: 13F55, 05E40, Secondary: 13C40,14M12. 
ideals, we can consider these monomial ideals to be in a finitely generated polynomial ring $\mathbb{k}\left[x_{S}\right]$.

Regular quotients. If $S \subseteq P \times \mathbb{N}$ is the support, an isotone map $\psi: S \rightarrow R$ gives a map of polynomial rings $\mathbb{k}\left[x_{S}\right] \rightarrow \mathbb{k}\left[x_{R}\right]$ given by dividing $\mathbb{k}\left[x_{S}\right]$ by a regular sequence consisting of differences $x_{s}-x_{s^{\prime}}$ where $\phi(s)=\phi\left(s^{\prime}\right)$. We give conditions, Theorems 2.1 and 2.2 , such that this is a regular sequence for $\mathbb{k}\left[x_{S}\right] / L(\mathcal{J}, P)$ resp. $\mathbb{k}\left[x_{S}\right] / L(P, \mathcal{J})$. This generalizes the main results of [6] where we by many examples show the omnipresence of letter- and co-letterplace ideals in the literature on monomial ideals.

$P$-stable ideals in $\mathbb{k}\left[x_{P}\right]$. In particular the projection $S \subseteq P \times \mathbb{N} \stackrel{p}{\longrightarrow} P$ fulfill these conditions for the letterplace ideal $L(\mathcal{J}, P)$. Hence we get ideals $L^{p}(\mathcal{J}, P) \subseteq \mathbb{k}\left[x_{P}\right]$ whose quotient ring is a regular quotient of $\mathbb{k}\left[x_{S}\right] / L(\mathcal{J}, P)$. When $P$ is the antichain these give all the monomial ideals in $\mathbb{k}\left[x_{P}\right]$, and $L(\mathcal{J}, P)$ is the standard polarization of the monomial ideal $L^{p}(\mathcal{J}, P) \subseteq \mathbb{k}\left[x_{P}\right]$. When $P=[m]$ is the chain, these give precisely the strongly stable ideals in $\mathbb{k}\left[x_{[m]}\right]$. For general $P$ we call such ideals $P$-stable and we give conditions for ideals to fulfill this notion. The ideals $L(\mathcal{J}, P)$ will then be non-standard polarizations of $L^{p}(\mathcal{J}, P)$. The notion of $P$-stable is not the same as the notion of $P$-Borel in [8]. Rather the notion of $P$-stable is more subtle. For instance a power of the graded maximal ideal $\mathfrak{m}^{n}$ in $\mathbb{k}\left[x_{P}\right]$, with $n \geq 2$, is $P$-stable iff $P$ is the disjoint union of rooted trees with the roots on top.

Strongly stable ideals. In case $P$ is the chain, we show the Alexander duality of $L(\mathcal{J}, P)$ and $L(P, \mathcal{J})$ induces a duality between strongly stable ideals in $\mathbb{k}\left[x_{1}, \ldots, x_{m}\right]$ and finitely generated $m$-regular strongly stable ideals in $\mathbb{k}\left[x_{\mathbb{N}}\right]$. In particular it gives a duality between $n$-regular strongly stable ideals in $\mathbb{k}\left[x_{1}, \ldots, x_{m}\right]$ and $m$-regular strongly stable ideals in $\mathbb{k}\left[x_{1}, \ldots, x_{n}\right]$, which seemingly has not been noticed before. These are joint results with Alessio D'Ali and Amin Nematbakhsh.

Principal poset ideals. A distinguished class of poset ideals of $\operatorname{Hom}(P, \mathbb{N})$ arises by considering an isotone map $\alpha: P \rightarrow \mathbb{N}$ and letting $\mathcal{J}$ be the set of all $\phi$ such that $\phi \leq \alpha$. These give the principal letterplace ideals $L(\alpha, P)$ and co-letterplace ideals $L(P, \alpha)$. The ideals $L(n, P)$ and $L(P, n)$ of [6] are the special cases when $\alpha$ is the constant map sending each $p$ to $n$. When $P$ is the antichain $L(\alpha, P)$ is a complete intersection, and these ideals may therefore be considered as generalizations of complete intersections.

Determinantal ideals. A particular case of principal letterplace ideals is when $P$ is the chain $[m]$. In [6] we showed that $L(n,[m])$ is the initial ideal of the ideal of maximal minors of an $(m+n-1) \times n$-matrix. Here we show that $L(\alpha,[m])$ is also an initial ideal of a class of determinantal ideals. This is a class which seemingly has not been considered before, and which in a natural way generalizes the determinantal ideals of maximal minors. All these ideals are Cohen-Macaulay of codimension $m=|P|$.

The organization of the paper is as follows. In Section 1 we define the (generalized) letterplace ideal $L(\mathcal{J}, P)$ and the co-letterplace ideal $L(P, \mathcal{J})$ and show they are Alexander dual. Section 2 gives the conditions ensuring that we divide out by a regular sequence of variable differences for the quotient rings. Section 3 gives the conditions for a monomial ideal in $\mathbb{k}\left[x_{P}\right]$ to be $P$-stable. Principal letterplace ideals are introduced in Section 4 and we show how the initial ideals of certain classes of determinantal ideals 
studied by W.Bruns and U.Vetter in [1] and J.Herzog and N.Trung in [1] are regular quotients of principal letterplace ideals. Section 5 gives the class of determinantal ideals generalizing ideals of maximal minors. In Section 6 we show the duality on strongly stable ideals, and the last Section 7 gives the proofs of the statements of Section 2 on regular sequences.

\section{The poset of isotone maps to $\mathbb{N}$}

We first give various notions and results concerning order preserving maps from a poset $P$ to the integers $\mathbb{N}$. Then we define letterplace and co-letterplace ideals in a more general setting than in the previous article [6].

1.1. Graphs and bases of isotone maps. If $P$ and $Q$ are posets, an isotone map $\phi: P \rightarrow Q$ is a map such that $p \leq p^{\prime}$ implies $\phi(p) \leq \phi\left(p^{\prime}\right)$. The set of isotone map is denoted $\operatorname{Hom}(P, Q)$ and is itself a poset with $\psi \leq \phi$ if $\psi(p) \leq \phi(p)$ for every $p \in P$. Thus the category of posets has an internal Hom. We also have the product poset $P \times Q$. This makes the category of posets into a symmetric monoidal closed category. A subset $J \subseteq Q$ is a poset ideal if $q \in J$ and $q^{\prime} \leq q$ implies $q^{\prime} \in J$. A subset $F \subseteq Q$ is a poset filter if $q \in F$ and $q \leq q^{\prime}$ implies $q^{\prime} \in F$. Note that $J$ is a poset ideal, iff its complement $J^{c}$ is a poset filter.

Denote by $\mathbb{N}=\{0,1,2, \cdots\}$ the natural numbers including 0 . Henceforth we shall always assume we have a finite partially ordered set $P$. We get the poset $\operatorname{Hom}(P, \mathbb{N})$.

Remark 1.1. $\operatorname{Hom}(P, \mathbb{N})$ is also a monoid coming from addition on $\mathbb{N}: \phi+\psi$ is the map sending $p$ to $\phi(p)+\psi(p)$. Denote by $\hat{P}=P \cup\{1\}$ where 1 is a maximal element, so $1>p$ for any $p \in P$. Then the semi-group $\operatorname{ring} \mathbb{k} \operatorname{Hom}(\hat{P}, \mathbb{N})$ is the Hibi ring of the distributive lattice $D(P)$ of order ideals in $P$. See V.Ene [3] for a survey on Hibi rings. The semi-group ring $\mathbb{k} \operatorname{Hom}(P, \mathbb{N})$ was first studied by A.Garcia in [9] and more recently by V.Feray and V.Reiner in [5]. We shall in this article only use the poset structure on $\operatorname{Hom}(P, \mathbb{N})$ and not the monoid structure.

Lemma 1.2 (Generalized Dickson's lemma). For a finite poset $P$, any subset $S$ of $\operatorname{Hom}(P, \mathbb{N})$ has a finite set of minimal elements.

Proof. Let $q \in P$ be a maximal element. The inclusion $P \backslash\{q\} \hookrightarrow P$ induces a projection $\operatorname{Hom}(P, \mathbb{N}) \rightarrow \operatorname{Hom}(P \backslash\{q\}, \mathbb{N})$. Let $T$ be the image of $S$ under this projection. By induction $T$ has a finite set of minimal elements (maps): $\alpha_{1}, \ldots, \alpha_{r}$. Consider the $\phi$ such that the restriction $\phi_{\mid P \backslash q}=\alpha_{i}$, and let $n_{i}$ be the minimal value of $\phi(q)$ for these $\phi$. Let $N$ be the maximum of the $n_{i}$. Each $\alpha_{i}$ extends to a map $\beta_{i} \in S$ with $\beta_{i}(q) \leq N$.

For $j \leq N$ let $S_{j} \subseteq S$ be the set of all $\phi \in S$ such that $\phi(q)=j$, and let $T_{j}$ be its projection onto $\operatorname{Hom}(P \backslash\{q\}, \mathbb{N})$. By induction, each $T_{j}$ for $j=1, \ldots, N$ has a finite set of minimal elements $T_{j}^{\mathrm{min}}$. Extending $T_{j}^{\mathrm{min}}$ naturally to $S$ we get a set $S_{j}^{\mathrm{min}} \subseteq S$

If now $\phi \in S$, then either $\phi(q) \geq N$, and so $\phi \geq \beta_{i}$ for some $\beta_{i}$, or $\phi(q)=j \leq N$ and then $\phi \geq \beta$ for some $\beta \in S_{j}^{\text {min }}$. Thus $S$ has a finite set of minimal elements.

Definition 1.3. Let $\phi: P \rightarrow \mathbb{N}$ be an isotone map. The graph of $\phi$ is

$$
\Gamma \phi=\{(p, i) \mid \phi(p)=i\} \text {. }
$$


The ascent of $\phi$ is

$$
\Lambda \phi=\{(p, i) \mid \phi(q) \leq i<\phi(p) \text { for all } q<p\} .
$$

Both the graph and the ascent are subsets of $P \times \mathbb{N}$.

Remark 1.4. The union $\Gamma \phi \cup \Lambda \phi$ is denoted $T_{*} \phi$ in [2, Section2]. The intervals [ $\left.\Gamma \phi, T_{*} \phi\right]$ index the resolution of the co-letterplace ideals $L(P, n ; \mathcal{J})$, see [2, Subsection 4.3]. This generalizes the Eliahou-Kervaire resolutions of strongly stable ideals generated in a single degree.

Lemma 1.5. Let $\mathcal{F} \subseteq \operatorname{Hom}(P, \mathbb{N})$ be a filter. The set of all ascents $\Lambda \phi$ where $\phi \in \mathcal{F}$, has a finite set of inclusion minimal elements, i.e. which are minimal for the partial order of inclusion on sets.

Proof. Let $N$ be the maximum of all values $\phi(p)$ as $\phi$ ranges over the minimal elements of $\mathcal{F}$ (which is a finite set by the previous Lemma 1.2) and $p$ ranges over $P$. Given an isotone map $\psi$ in $\mathcal{F}$, let

$$
n=\max \psi=\max \{\psi(p) \mid p \in P\} .
$$

If $n>N$ we want to show that $\Lambda \psi$ is not inclusion minimal among $\Lambda \phi$ where $\phi \in \mathcal{F}$. Then any inclusion minimal $\Lambda \phi$ for $\phi \in \mathcal{F}$ will have $\phi(p) \leq N$ for all $p \in P$, and so there is only a finite number of such $\phi$.

So suppose $n>N$. Then $\psi$ is not minimal in $\mathcal{F}$. Let $F \subseteq P$ be the poset filter of all $p \in P$ with $\psi(p)=n$, and $F_{\min }$ the minimal elements in $F$. Then the map

$$
\phi(p)=\left\{\begin{array}{ll}
\psi(p) & p \notin F \\
n-1 & p \in F
\end{array},\right.
$$

is also in $\mathcal{F}$. But then clearly $\Lambda \phi=\Lambda \psi \backslash\left\{(p, n-1) \mid p \in F_{\min }\right\}$. This proves the statement.

Definition 1.6. Let $\mathcal{J} \subseteq \operatorname{Hom}(P, \mathbb{N})$ be a poset ideal. A marker for $\mathcal{J}$ is given by a poset ideal $I \subseteq P$ and an isotone map $\alpha: I \rightarrow \mathbb{N}$ such that every isotone map $\phi: P \rightarrow \mathbb{N}$ with restriction $\phi_{\mid I}=\alpha$, is in $\mathcal{J}$. It is a minimal marker if no restriction $\alpha_{\mid J}$ to a poset ideal $J$ properly contained in $I$, is a marker for $\mathcal{J}$. This means that the graph of the marker $\alpha$ is inclusion minimal among graphs of markers for $\mathcal{J}$.

Remark 1.7. When $P$ is an antichain, the notion of poset ideal in $\operatorname{Hom}(P, \mathbb{N})$ is close to the notion of multicomplex studied in [10, Section 9].

Proposition 1.8. Let $\mathcal{J} \subseteq \operatorname{Hom}(P, \mathbb{N})$ be a poset ideal. The (unique) set of minimal markers is a finite set.

Proof. We first consider minimal markers $\phi: P \rightarrow \mathbb{N}$, i.e. minimal markers whose domain is $P$. Let $\mathcal{M}$ be the set of such minimal markers. Suppose $\mathcal{M}$ is infinite. Then there is some maximal $q \in P$ such that $\phi(q)$ may become arbitrarily large for $\phi \in \mathcal{M}$. If the set $\mathcal{M}_{q}$ of restrictions $\phi_{\mid P \backslash\{q\}}$ where $\phi \in \mathcal{M}$ is finite, then clearly one of these restricted maps is a marker, contradicting that $\phi$ is a minimal marker. So the set $\mathcal{M}_{q}$ is infinite. But by the above Proposition 1.2 it contains a finite set of minimal elements. 
Then for at least one of these minimal elements, $\alpha$, there is an infinite number of $\phi \in \mathcal{M}$ such that $\phi_{\mid P \backslash\{q\}} \geq \alpha$ and $\phi(q)$ is arbitrarily large. But since $\mathcal{J}$ is a poset ideal, there must then be $\phi: P \rightarrow \mathbb{N}$ with $\phi_{\mid P \backslash\{q\}}=\alpha$ and $\phi(q)$ arbitrarily large, contradicting that $\mathcal{M}$ consists of minimal markers.

Hence the set of inclusion minimal markers whose domain is $P$, is finite. Let $I$ be a poset ideal contained in $P$. Let $\mathcal{J}_{\mid I}$ be the poset ideal of $\operatorname{Hom}(I, \mathbb{N})$ consisting of markers supported on $I$. By the argument as above, there is a finite number of minimal markers whose domain is $I$. Since there is only a finite number of such $I$ there will be only a finite number of minimal markers for $\mathcal{J}$.

1.2. Letterplace and co-letterplace ideals. For a set $S$ let $\mathbb{k}\left[x_{S}\right]$ be the polynomial ring over a field $\mathbb{k}$ in the variables $x_{s}$ where $s \in S$. We shall in particular consider the polynomial ring $\mathbb{k}\left[x_{P \times \mathbb{N}}\right]$, a polynomial ring in the infinite number of variables $x_{p, i}$ where $p \in P, i \in \mathbb{N}$.

Definition 1.9. Let $\mathcal{J} \subseteq \operatorname{Hom}(P, \mathbb{N})$ be a poset ideal. The co-letterplace ideal $L(P, \mathcal{J})$ is the monomial ideal in $\mathbb{k}\left[x_{P \times \mathbb{N}}\right]$ generated by the monomials $m_{\Gamma \alpha}$ associated to the graphs of markers $\alpha$ of $\mathcal{J}$. It is clearly the same as the ideal generated by the finite set of monomials associated to graphs of minimal markers.

The letterplace ideal $L(\mathcal{J}, P)$ is the monomial ideal in $\mathbb{k}\left[x_{P \times \mathbb{N}}\right]$ generated by the monomials $m_{\Lambda \phi}$ associated to ascents of the isotone maps $\phi$ in the complement filter $\mathcal{J}^{c}$. It is clearly the same as the ideal generated by the finite set of monomials of inclusion minimal ascents.

Remark 1.10. Let $[n]=\{1<2<\cdots<n\}$. In [6] the setting was a poset ideal $\mathcal{J} \subseteq \operatorname{Hom}(P,[n])$ and we defined letterplace and co-letterplace ideals $L(n, P ; \mathcal{J})$ and $L(P, n ; \mathcal{J})$ in the polynomial ring $\mathbb{k}\left[x_{P \times[n]}\right]$. This corresponds to finite poset ideals $\mathcal{J}$ in the definition above, see Section 4. In [2] we showed that the Stanley-Reisner ideal $L(n, P ; \mathcal{J})$ defines a simplicial ball and described precisely the Gorenstein ideal defining its boundary.

Recall that for two squarefree monomial ideals $I$ and $J$ in a polynomial ring $S$ then $I$ is said to be Alexander dual to $J$ if the monomials in $I$ are precisely the monomials in $S$ which have nontrivial common divisor with every monomial in $J$. Then $J$ will also be Alexander dual to $I$.

The following generalizes [4, Theorem 1.1] and [6, Prop.1.2].

Proposition 1.11. The letterplace ideal $L(\mathcal{J}, P)$ and the co-letterplace ideal $L(P, \mathcal{J})$ are Alexander dual.

Our proof is close to the proof of Theorem 5.9 of [6]. Let us first recall some lemmata. Let $\mathcal{J}$ be a poset ideal in $\operatorname{Hom}(P, \mathbb{N})$ and $\mathcal{J}^{c}$ its complement filter. The following is Lemma 5.7 in [6].

Lemma 1.12. Let $\phi \in \mathcal{J}$ and $\psi \in \mathcal{J}^{c}$. Then $\Gamma \phi \cap \Lambda \psi$ is nonempty.

The following is Lemma 5.8 in [6].

Lemma 1.13. Given a subset $S$ of $P \times \mathbb{N}$. Suppose it is disjoint from $\Gamma \phi$ for some $\phi$ in $\operatorname{Hom}(P, \mathbb{N})$. If $\phi$ is minimal such w.r.t. the partial order on $\operatorname{Hom}(P, \mathbb{N})$, then $S \supseteq \Lambda \phi$. 
Proof of Proposition 1.11. We show the following:

1. The letterplace ideal $L(\mathcal{J}, P)$ is contained in the Alexander dual of $L(P, \mathcal{J})$ : Every monomial in $L(\mathcal{J}, P)$ has non-trivial common divisor with every monomial in $L(P, \mathcal{J})$. 2. The Alexander dual of $L(P, \mathcal{J})$ is contained in the letterplace ideal $L(\mathcal{J}, P)$ : If a finite $S \subseteq P \times \mathbb{N}$ intersects every $\Gamma \alpha$ where $\alpha: I \rightarrow \mathbb{N}$ is a marker for $\mathcal{J}$, the monomial $m_{S}$ is in $L(\mathcal{J}, P)$.

1. Let $\alpha: I \rightarrow \mathbb{N}$ be a marker for $\mathcal{J}$ and let $\psi \in \mathcal{J}^{c}$. By Lemma 1.12, for every extension $\phi$ of $\alpha, \Gamma \phi$ and $\Lambda \psi$ intersect nonempty. But then clearly $\Gamma \alpha$ and $\Lambda \psi$ intersect nonempty.

2. Suppose a finite $S$ intersects every $\Gamma \alpha$ where $\alpha: I \rightarrow \mathbb{N}$ is a marker for $\mathcal{J}$. Then $S$ intersects every $\Gamma \phi$ where $\phi \in \mathcal{J}$. Let $\psi$ be a minimal element in $\operatorname{Hom}(P, \mathbb{N})$ such that $\Gamma \psi$ and $S$ intersect empty. Then $\psi \in \mathcal{J}^{c}$ and by Lemma $1.13 S \supseteq \Lambda \psi$. Therefore the monomial $m_{S}$ is in $L(\mathcal{J}, P)$.

When $I$ and $J$ are two Alexander dual monomial ideals, their set of minimal generators will involve exactly the same variables. The support $\operatorname{Supp}(\mathcal{J})$ of the poset ideal $\mathcal{J}$ of $\operatorname{Hom}(P, \mathbb{N})$ is the set pairs $(p, i)$ such that $x_{p, i}$ is a variable in one of the minimal generators of $L(P, \mathcal{J})$, or equivalently $L(\mathcal{J}, P)$. This is a finite set by the minimality observations in Definition 1.9. We can then consider the letterplace and co-letterplace ideals $L(P, \mathcal{J})$ and $L(\mathcal{J}, P)$ to live in the ring $\mathbb{k}\left[x_{\operatorname{Supp}(\mathcal{J})}\right]$, or in any polynomial ring $\mathbb{k}\left[x_{S}\right]$ where $\operatorname{Supp}(\mathcal{J}) \subseteq S \subseteq P \times \mathbb{N}$. Most of our statements will be independent of what the ambient ring is. In general the set $\operatorname{Supp}(\mathcal{J})$ is not so easily described, but in the case when $\mathcal{J}$ is finite there is a nice description, Section 4 .

\section{Regular QuOtients}

In [6, Section 3] we gave many examples of ideals which derive from letterplace ideals, and in [6, Section 6] many examples of ideals which derive from co-letterplace ideals. But the point is that they derive from them by cutting down the corresponding quotient ring by a regular sequence. The conditions ensuring that we have a regular sequence, are the main technical results of [6]. Here we generalize these results to the setting of any poset ideal in $\operatorname{Hom}(P, \mathbb{N})$.

2.1. Regular sequences. A subset $S$ of $P \times \mathbb{N}$ gets the induced poset structure. Let $S \stackrel{\phi}{\longrightarrow} R$ be an isotone map. Denote by $P^{\text {op }}$ be the opposite poset of $P$, i.e. $p \leq_{\text {op }} q$ if $p \geq q$. We say that $\phi$ has right strict chain fibers if $\phi^{-1}(r) \subseteq S$ is a chain when considered in $P^{\mathrm{op}} \times \mathbb{N}$, and all elements in this chain have distinct second coordinate. So if $(p, i)$ and $(q, j)$ are distinct elements in the fiber with $i \leq j$, then $i<j$ and $p \geq q$ for the partial order on $P$.

Given a poset ideal $\mathcal{J} \subseteq \operatorname{Hom}(P, \mathbb{N})$, choose a finite $S$ such that $\operatorname{Supp}(\mathcal{J}) \subseteq S \subseteq$

$P \times \mathbb{N}$. Any map $S \stackrel{\phi}{\longrightarrow} R$ gives a map of linear spaces $\left\langle x_{S}\right\rangle \stackrel{\tilde{\phi}}{\longrightarrow}\left\langle x_{R}\right\rangle$. Let $B$ be a basis for the kernel of $\tilde{\phi}$, whose elements are differences $x_{p, i}-x_{q, j}$ such that $\phi(p, i)=\phi(q, j)$. 
Theorem 2.1. Given a poset ideal $\mathcal{J} \subseteq \operatorname{Hom}(P, \mathbb{N})$, and let $\operatorname{Supp}(\mathcal{J}) \subseteq S \subseteq P \times \mathbb{N}$ with $S$ finite. Let $\phi: S \rightarrow R$ be an isotone map with right strict chain fibers. Then the basis $B$ is a regular sequence for $\mathbb{k}\left[x_{S}\right] / L(\mathcal{J}, P)$.

We prove this in Section 7. It is a generalization of Theorem 5.6 in 6]. Its proof follows rather closely the proof in [6] but with some new elements. We write $L^{\phi}(\mathcal{J}, P)$ for the ideal in $\mathbb{k}\left[x_{R}\right]$ generated by the image of $L(\mathcal{J}, P)$ by $\phi$.

Consider again an isotone map $S \stackrel{\phi}{\longrightarrow} R$ as above. We say it has left strict chain fibers if $\phi^{-1}(r) \subseteq S$ is a chain when considered in $P^{\mathrm{op}} \times \mathbb{N}$ and all its elements have distinct first coordinates.

Theorem 2.2. Given a poset ideal $\mathcal{J} \subseteq \operatorname{Hom}(P, \mathbb{N})$, and let $\operatorname{Supp}(\mathcal{J}) \subseteq S \subseteq P \times \mathbb{N}$ with $S$ finite. Let $\phi: S \rightarrow R$ be an isotone map with left strict chain fibers. Then the basis $B$ is a regular sequence for $\mathbb{k}\left[x_{S}\right] / L(P, \mathcal{J})$.

Again we prove this in Section 7, and it is a generalization of Theorem 5.12 in [6]. Its proof also follows rather closely the proof in [6]. We write $L^{\phi}(P, \mathcal{J})$ for the ideal in $\mathbb{k}\left[x_{R}\right]$ generated by the image of $L(P, \mathcal{J})$ by $\phi$.

\section{Monomial ideals in $\mathbb{k}\left[x_{P}\right]$}

When $P$ is the antichain on $m$ elements, then $\operatorname{Hom}(P, \mathbb{N})$ identifies as $\mathbb{N}^{m}$. A poset filter $\mathcal{J}^{c}$ in $\mathbb{N}^{m}$ corresponds naturally to a monomial ideal in $\mathbb{k}\left[x_{1}, \ldots, x_{m}\right]$. In this case the letterplace ideal $L(\mathcal{J}, P)$ is the standard polarization of this monomial ideal. In this section we associate monomial ideals in $\mathbb{k}\left[x_{P}\right]$ to poset filters $\mathcal{J}^{c} \in \operatorname{Hom}(P, \mathbb{N})$ for any poset $P$. The extra structure added in this setting, is that the letterplace ideal $L(\mathcal{J}, P)$ may be considered a (non-standard) polarization of the monomial ideal in $\mathbb{k}\left[x_{P}\right]$.

Let $p: P \times \mathbb{N} \rightarrow P$ be the projection map onto the first coordinate $P$. This map has right strict chain fibers, and so for any poset ideal $\mathcal{J} \subseteq P \times \mathbb{N}$ we get by Theorem 2.1 an ideal $L^{p}(\mathcal{J}, P)$ in $\mathbb{k}\left[x_{P}\right]$ whose quotient ring is a regular quotient of the quotient ring $\mathbb{k}\left[x_{S}\right] / L(\mathcal{J}, P)$, for suitable finite $S \subseteq P \times \mathbb{N}$.

In the first subsection we get a direct description of the ideal $L^{p}(\mathcal{J}, P)$ and its quotient ring in terms of the poset ideal $\mathcal{J}$. In the next subsection we achieve an intrinsic description of which monomial ideals in $\mathbb{k}\left[x_{P}\right]$ that come from a poset ideal $\mathcal{J}$ in $\operatorname{Hom}(P, \mathbb{N})$.

3.1. Correspondence between monomials in $\mathbb{k}\left[x_{P}\right]$ and elements of $\operatorname{Hom}(P, \mathbb{N})$. Given a poset $P$. The polynomial ring $\mathbb{k}\left[x_{P}\right]$ may be identified as the semi-group ring of the monoid $\mathbb{N} P$ consisting of sums $\sum_{p \in P} n_{p} p$ where $n_{p} \in \mathbb{N}$. This monoid is naturally also a poset by $\sum_{p \in P} n_{p} p \leq \sum_{p \in P} m_{p} p$ if each $n_{p} \leq m_{p}$ (so this poset structure is determined only by the cardinality of $P$ ). The monoid $\mathbb{N} P$ naturally identifies as the monomials in $\mathbb{k}\left[x_{P}\right]$ and we shall freely use this identification.

Now given $\phi \in \operatorname{Hom}(P, \mathbb{N})$. The ascent $\Lambda \phi \subseteq P \times \mathbb{N}$. Taking the formal sum of its elements, we consider it as an element of the monoid $\mathbb{N}(P \times \mathbb{N})$. By the projection $P \times \mathbb{N} \rightarrow P$ we get the map of monoids $\mathbb{N}(P \times \mathbb{N}) \rightarrow \mathbb{N} P$. Denote by $\bar{\Lambda} \phi$ the image of $\Lambda \phi$ by this map. 
Example 3.1. Consider the poset $P$ below and the map $\phi: P \rightarrow \mathbb{N}$ sending the vertices to the numbers to the right.

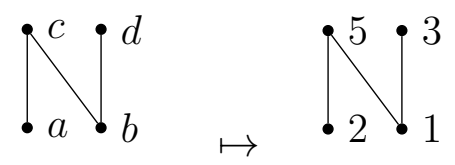

Then $\bar{\Lambda} \phi$ is the monomial $x_{a}^{2} x_{b} x_{c}^{3} x_{d}^{2}$.

For antichains $A$ and $B$ in $P$ define an order relation by $A \leq B$ if for every $b \in B$ there is an $a \in A$ with $a \leq b$ (but for $a \in A$ we do not require there to be $b \in B$ with $a \leq b)$. If $A$ and $B$ generate the filters $F_{A}$ and $F_{B}$, this corresponds precisely to $F_{A} \supseteq F_{B}$.

Given an isotone map

$$
\phi: P \rightarrow \mathbb{N}
$$

we get a filter $F_{m}=\phi^{-1}[m,+\infty\rangle$ and a descending chain

$$
P=F_{0} \supseteq F_{1} \supseteq F_{2} \supseteq \cdots
$$

of poset filters. Let $A_{i}=\min F_{i}$. This is an antichain and the $A_{i}$ fulfill the order relation given above

$$
A_{0} \leq A_{1} \leq A_{2} \leq \cdots .
$$

Lemma 3.2. There are one-to-one correspondences between isotone maps (11), chains of poset filters (2), and chains of antichains (3). Furthermore:

a. $(p, i) \in \Lambda \phi$ iff $p \in A_{i+1}$

b. The projection $\bar{\Lambda} \phi$ is $\sum_{i>0} A_{i}$ considered as an element of $\mathbb{N} P$.

Proof. The one-to-one correspondences are clear. That $(p, i) \in \Lambda \phi$ means $i<\phi(p)$ and $\phi(q) \leq i$ for every $q<p$. Then $p \in F_{i+1}$ and since $q$ is not in $F_{i+1}$ we get $p \in A_{i+1}$. Conversely if $p \in A_{i+1}$, then $\phi(p) \geq i+1$ and when $q<p$ then $q \notin F_{i+1}$ and so $\phi(q) \leq i$. Thus $(p, i)$ in $\Lambda \phi$.

For statement b. let $\phi(p)=r$ and $s=\max \{\phi(q) \mid q<p\}$, so $\Lambda \phi$ contains $\{(p, s),(p, s+$ $1), \ldots,(p, r-1)\}$ and these are all the elements with $p$ as first coordinate. Then $\bar{\Lambda} \phi=\cdots+(r-s) p+\cdots$. But $p$ is then in precisely $A_{s+1}, \cdots, A_{r}$ and so $\sum_{i>0} A_{i}=$ $\cdots+(r-s) p+\cdots$.

Proposition 3.3. The map

$$
\bar{\Lambda}: \operatorname{Hom}(P, \mathbb{N}) \rightarrow \mathbb{N} P
$$

is a bijection.

Proof. Given an isotone $\phi: P \rightarrow \mathbb{N}$ we get by the above lemma a filtration of poset filters

$$
P=F_{0} \supseteq F_{1} \supseteq \cdots \supseteq F_{N}=\emptyset \text {. }
$$

Injectivity of $\bar{\Lambda}$ : If $\phi \neq \psi$, then $F_{i}^{\phi} \neq F_{i}^{\psi}$ for some $i$. Let $i$ be minimal such. Then for say $\phi$ there is a minimal $p \in F_{i}^{\phi}$ such that no $q \in F_{i}^{\psi}$ is $\leq p$. Then $\bar{\Lambda} \phi=\cdots+j_{1} p+\cdots$ and $\bar{\Lambda} \psi=\cdots+j_{2} p+\cdots$ where $j_{2}<j_{1}$ (since $i$ is minimal) and so $\bar{\Lambda} \phi \neq \bar{\Lambda}(\psi)$. 
Surjectivity of $\bar{\Lambda}$ : Given $\mathbf{a}_{1}=\sum a_{1 p} p \in \mathbb{N} P$. Let $A_{1}$ be the set of minimal elements of $\left\{p \mid a_{1 p} \neq 0\right\}$ and $F_{1}$ the filter generated by $A_{1}$. Considering the set $A_{1}$ as a formal sum in $\mathbb{N} P$, let $\mathbf{a}_{2}=\mathbf{a}_{1}-A_{1}$ and let $F_{2}$ be the filter generated by $A_{2}$, the set of minimal elements $\min \left\{p \mid a_{2 p} \neq 0\right\}$. Continuing we get a sequence

$$
P=F_{0} \supseteq F_{1} \supseteq \cdots \supseteq F_{N}=\emptyset .
$$

This determines a map $\phi$ such that $\bar{\Lambda} \phi=\mathbf{a}_{1}$.

Proposition 3.4. If $\mathcal{J}$ is a poset ideal in $\operatorname{Hom}(P, \mathbb{N})$, then $\bar{\Lambda} \mathcal{J}$ is a poset ideal in $\mathbb{N} P$.

Proof. Let $A_{1} \leq A_{2}$ be antichains, and $D_{1} \subseteq A_{1}$. Let $D_{2}$ be the largest subset of $A_{2}$ such that $B_{1}=\left(A_{1} \backslash D_{1}\right) \cup D_{2}$ is an antichain. (Note that there is a unique maximal such $D_{2}$.) Let $B_{2}=A_{2} \backslash D_{2}$. Then $B_{1} \leq B_{2}$ are antichains, $A_{1} \leq B_{1}$ and $A_{2} \leq B_{2}$, and $A_{1}+A_{2}-D_{1}=B_{1}+B_{2}$ in $\mathbb{N} P$. If we have a chain $A_{1} \leq A_{2} \leq A_{3}$ we may let $B_{2}=\left(A_{2} \backslash D_{2}\right) \cup D_{3}$ and $B_{3}=A_{3} \backslash D_{3}$. Again we have $A_{3} \leq B_{3}$ and $A_{1}+A_{2}+A_{3}-D_{1}$ equals $B_{1}+B_{2}+B_{3}$. In this way we may continue if we have longer chains.

Now given $\phi \in \mathcal{J}$. Let $n=\max _{p \in P} \phi(p)$. Then $\phi$ corresponds to a chain, with $A_{i}=\min F_{i}$ in (4):

$$
A_{1} \leq A_{2} \leq \cdots \leq A_{n}
$$

Furthermore $\bar{\Lambda}(\phi)=\sum_{i=1}^{n} A_{i}$. Let $p \in A_{i}$. We show that $\bar{\Lambda} \phi-p$ is also in $\bar{\Lambda} \mathcal{J}$, proving that $\bar{\Lambda} \mathcal{J}$ is also a poset ideal. If we remove a $p \in A_{i}$ from this, the above procedure gives a chain

$$
B_{1} \leq B_{2} \leq \cdots \leq B_{n}
$$

where $B_{j}=A_{j}$ for $j \leq i-1$ and $B_{i}=A_{i} \backslash\{p\}$. Also $A_{i} \leq B_{i}$ for every $i$ and so this chain corresponds to an isotone map $\psi$ with $\psi \leq \phi$ and with

$$
\bar{\Lambda} \psi=\sum_{i} B_{i}=\left(\sum_{i} A_{i}\right)-p=\bar{\Lambda} \phi-p
$$

and so the latter is in $\bar{\Lambda} \mathcal{J}$.

Remark 3.5. It is not true in general that if $Q \rightarrow P$ is a bijective isotone map, so $Q$ is a weakening of the partial order on $P$, the natural bijection

$$
\bar{\Lambda}_{Q}^{-1} \circ \bar{\Lambda}_{P}: \operatorname{Hom}(P, \mathbb{N}) \rightarrow \operatorname{Hom}(Q, \mathbb{N})
$$

takes poset ideals to poset ideals.

Now we can round off with our goal of getting a description of $L^{p}(\mathcal{J}, P)$ in $\mathbb{k}\left[x_{P}\right]$ where $p: P \times \mathbb{N} \rightarrow P$ is the projection map.

Corollary 3.6. The set of monomials in $L^{p}(\mathcal{J}, P)$ is precisely the image by $\bar{\Lambda}$ of the filter $\mathcal{J}^{c}$. In other words, the normal (i.e. nonzero) monomials in $\mathbb{k}\left[x_{P}\right] / L^{p}(\mathcal{J}, P$ ) are precisely the $\bar{\Lambda} \phi$ for $\phi \in \mathcal{J}$.

Proof. $L(\mathcal{J}, P)$ is generated by $\Lambda \phi$ for $\phi \in \mathcal{J}^{c}$, so $L^{p}(\mathcal{J}, P)$ is generated by the $\bar{\Lambda} \phi$ for $\phi \in \mathcal{J}^{c}$. But the image of $\mathcal{J}^{c}$ by $\bar{\Lambda}$ is a poset filter in $\mathbb{N} P$ or equivalently a monomial ideal in $\mathbb{k}\left[x_{P}\right]$. Hence the image is precisely $L^{p}(\mathcal{J}, P)$. 
Example 3.7. If $P$ is an antichain $\underline{m}=\{1,2, \ldots, m\}$, the map $\bar{\Lambda}$ of Proposition 3.3 is an isomorphism of posets. Hence in this case we get a one-to-one correspondence between poset filters in $\operatorname{Hom}(\underline{m}, \mathbb{N})$ and monomial ideals in $\mathbb{k}\left[x_{\underline{m}}\right]$, given by the map $\bar{\Lambda}$. The ascent $\Lambda$ sends the filter $\mathcal{J}^{c}$ to the letterplace ideal $L(\mathcal{J}, \bar{P})$ in $\mathbb{k}\left[x_{P \times \mathbb{N}}\right]$, which is a squarefree monomial ideal. This ideal is the standard polarization of the monomial ideal $L^{p}(\mathcal{J}, P)$ in $\mathbb{k}\left[x_{P}\right]$, whose normal monomials are precisely $\bar{\Lambda} \mathcal{J}$.

In Section 6 we show that the image of $\bar{\Lambda}$ when $P$ is a chain, is precisely the strongly stable ideals in $\mathbb{k}\left[x_{P}\right]$.

3.2. Which monomial ideals in $\mathbb{k}\left[x_{P}\right]$ come from $\operatorname{Hom}(P, \mathbb{N})$ ? Let $I \subseteq \mathbb{k}\left[x_{P}\right]$ be a monomial ideal. We want to investigate when $I=\bar{\Lambda} \mathcal{J}$ for a poset ideal $\mathcal{J} \subseteq \operatorname{Hom}(P, \mathbb{N})$. First we need some definitions.

Definition 3.8. Given $b \in P$, a multichain

$$
C: p_{1} \leq p_{2} \leq \cdots \leq p_{r}
$$

in $P$ is a $b$-chain if $p_{r} \leq b$. The associated monomial $m_{C}$ is $\prod_{i=1}^{r} x_{p_{i}}$. The $b$-chain goes through $a$ if $a \leq b$ and $a$ may be inserted in $C$ to make it a larger multichain (so $a$ may or may not be equal to one of the $p_{i}$ 's).

The $b$-chain $C$ is in a monomial $m$ if $m_{C}$ divides $m$. The $b$-chain $C$ is a longest $b$-chain in $m$ if there is no $b$-chain $C^{\prime}$ of $m$ with cardinality $\left|C^{\prime}\right|>|C|$. Note that there may be several longest $b$-chains of $m$.

Lemma 3.9. If $m=\bar{\Lambda} \phi$ and $b \in P$, the longest $b$-chain in $m$ has length $\phi(b)$.

Proof. Suppose $(p, i)$ and $(q, j)$ are distinct elements in $\Lambda \phi$ with $p \leq q \leq b$. If $p<q$ then $i<\phi(p) \leq j<\phi(q) \leq \phi(b)$. If $p=q$ we may assume $i<j$ and we have $j<\phi(p) \leq \phi(b)$. Thus in both cases $i<j<\phi(b)$. The consequence of this is that if $p_{1} \leq p_{2} \leq \cdots \leq p_{r}(\leq b)$ is a $b$-chain in $m$, then $r \leq \phi(b)$.

We now show that there is a $b$-chain in $m$ of length $r=\phi(b)$. If $b$ is minimal then

$$
m=\bar{\Lambda} \phi=r \cdot b+\text { other terms , }
$$

and so clearly $b \leq b \leq \cdots \leq b$ repeated $r$ times is a longest $b$-chain in $m$.

Suppose $b$ is not minimal and let $q<b$ be such that $s=\phi(q)$ is maximal. By induction on the height of elements of $P$ we may assume that there is a $q$-chain $p_{1} \leq \cdots \leq p_{s}$ in $m$. Since $(b, s), \ldots,(b, r-1)$ is in $\Lambda \phi$, this extends to a $b$-chain

$$
p_{1} \leq \cdots \leq p_{s} \leq b \leq \cdots \leq b
$$

in $m$, with $b$ repeated $r-s$ times. This chain has length $r=\phi(b)$.

Definition 3.10. An ideal $I \subseteq \mathbb{k}\left[x_{P}\right]$ is $P$-stable if the following holds: Let $m=n m_{B}$ be a monomial in $I$ where $B=\left\{b_{1}, \ldots, b_{r}\right\}$ is an antichain in $P$. Let $a \in P$ and suppose for every $b \in B$ there is a longest $b$-chain in $m$ going through $a$. Then $n \cdot x_{a} \in I$.

Proposition 3.11. An ideal $I \subseteq \mathbb{k}\left[x_{P}\right]$ is an image $I=\bar{\Lambda} \mathcal{J}^{c}$ for some poset filter $\mathcal{J}^{c} \subseteq \operatorname{Hom}(P, \mathbb{N})$ iff $I$ is P-stable.

We prove this after the remark and the example. 
Remark 3.12. If $a<b$ and $x_{b}$ divides $m$, we cannot say whether $\frac{m x_{a}}{x_{b}} \in I$. Only if there is a longest $b$-chain going through $a$ we can say this. Our notion of $P$-stable is therefore quite distinct from the notion of $P$-Borel in [8].

Example 3.13. Let $P$ be the poset

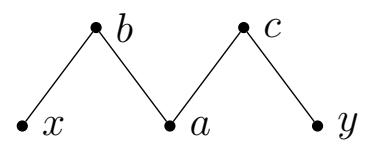

and $m=x^{4} a^{2} y^{3} b c^{2}$ (we write $p$ instead of $x_{p}$ for the variables). The only longest $b$-chain in $m$ is $x \leq x \leq x \leq x \leq b$. If $m$ is in a $P$-stable ideal $I$ then $\frac{m x}{b} \in I$. There is no longest $b$-chain through $a$, so even though $a<b$ we do not need to have $\frac{m a}{b} \in I$.

Let $P$ be

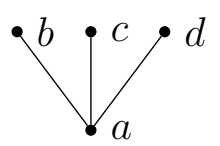

and $m=a b^{2} c^{3} d^{2}$. Then each of $b, c, d$ have longest chains in $m$ through $a$. Thus if $m$ is in a $P$-stable ideal $I$ then, letting $B=\{b, c, d\}$

Similarly

$$
m^{\prime}=\frac{m a}{b c d}=a^{2} b c^{2} d \in I \text {. }
$$

$$
\frac{m^{\prime} a}{b c d}=a^{3} c \in I
$$

Remark 3.14. When $P$ is a chain $[m]=\{1<2<\cdots<m\}$, an ideal $I \subseteq \mathbb{k}\left[x_{[m]}\right]$ is $P$ stable iff it is strongly stable, see Section 6 . When $P$ is an antichain $\underline{m}=\{1,2, \ldots, m\}$ i.e. with no distinct comparables, then any monomial ideal $I \subseteq \mathbb{k}\left[x_{\underline{m}}\right]$ is $P$-stable.

Proof of Proposition 3.11. We will first show that if $I$ is $\bar{\Lambda} \mathcal{J}^{c}$ for some ideal $\mathcal{J} \subseteq$ $\operatorname{Hom}(P, \mathbb{N})$, then $I$ is $P$-stable. We divide into two parts. Recall that if $q>p$ and there are no $r$ with $q>r>p$, then $q$ is said to cover $p$. Let $m$ be a monomial in $I$. By Proposition $3.3 m=\bar{\Lambda} \psi$ for some $\psi \in \operatorname{Hom}(P, \mathbb{N})$. Let $m=m_{B} \cdot n$ where $B$ is an antichain in $P$ and $a$ an element of $P$ such that for every $b \in B$ there is a longest $b$-chain through $a$.

1. In the first part, let each of the elements of the set $B$ cover $a$. For $b \in B$ a longest $b$-chain in $m$ through $a$, is then longer than a a longest $a$-chain in $m$, and so $\psi(b)>\psi(a)$. Let $C \supseteq B$ be the set of all covers of $a$.

Let $C^{\prime} \subseteq C$ be the $c \in C$ such that $\psi(c)=\psi(a)$. Then a longest $c$-chain and a longest $a$-chain in $m$ have the same length. Also $C^{\prime}$ is disjoint from $B$. Let $B^{\prime}=B \cup C^{\prime}$, let $m^{\prime}=m \cdot m_{C^{\prime}}$ and let $m^{\prime}$ correspond to $\psi^{\prime}$ by $\bar{\Lambda}$. For $c \in C^{\prime}$ the longest $c$-chain in $m^{\prime}$ is one more than the longest $a$-chain in $m^{\prime}$. So $\psi^{\prime}(c)=\psi(a)+1$. For $c \in\left(C \backslash C^{\prime}\right) \cup B$ clearly $\psi(c)>\psi(a)$ and so $\psi^{\prime}(c)>\psi(a)=\psi^{\prime}(a)$. Thus $\psi^{\prime}(c)>\psi^{\prime}(a)$ for every $c$ covering $a$. Define $\psi^{\prime \prime}$ by

$$
\psi^{\prime \prime}(p)= \begin{cases}\psi^{\prime}(p), & p \neq a \\ \psi^{\prime}(a)+1, & p=a\end{cases}
$$


Thus $\psi^{\prime \prime}$ is an isotone map covering $\psi^{\prime}$, and $\psi^{\prime}$ corresponds to $m^{\prime}=n \cdot m_{B} \cdot m_{C^{\prime}}$ and $\psi^{\prime \prime}$ corresponds to $m^{\prime \prime}=n x_{a}$.

2. In the second part we now induct on the longest chain from any $b \in B$ to $a$. Let $a_{1}, \ldots, a_{r}$ be the elements covering $a$. Then for every $b \in B$ there is a longest $b$-chain through some $a_{i}$ and $a$. Partition $B=B_{1} \cup \cdots \cup B_{r}$ such that the elements in $B_{i}$ have a longest $b$-chain through $a_{i}$ and $a$. By induction $n x_{a_{1}} m_{B_{2}} \cdots m_{B_{r}} \in I$ and $n x_{a_{1}} x_{a_{2}} m_{B_{3}} \cdots m_{B_{r}} \in I$ and in the end $n x_{a_{1}} \cdots x_{a_{r}} \in I$. By part 1 . above we now get $n x_{a} \in I$.

We now show that if $I$ is $P$-stable, then $I=\bar{\Lambda} \mathcal{J}^{c}$ for some poset ideal $\mathcal{J} \subseteq \operatorname{Hom}(P, \mathbb{N})$. By Proposition 3.3, $I=\bar{\Lambda} \mathcal{F}$ for some subset $\mathcal{F} \subseteq \operatorname{Hom}(P, \mathbb{N})$. We want to show that $\mathcal{F}$ is a filter. Given $\psi \in \mathcal{F}$ and $\tilde{\psi}>\psi$. Choose a maximal $a$ such that $\tilde{\psi}(a)>\psi(a)$. Define $\psi^{\prime}$ by

$$
\psi^{\prime}(p)= \begin{cases}\psi(p), & p \neq a \\ \psi(a)+1, & p=a\end{cases}
$$

Then $\psi^{\prime}$ is an isotone map. Let $C \subseteq P$ be the elements $c$ covering $a$, so $\psi(a)<\psi(c)$ for $c \in C$ (by definition of $a$ ). Let $C^{\prime} \subseteq C$ be the subset with a longest chain in $m$ through $a$. If $m=\bar{\Lambda} \psi$ then $\bar{\Lambda} \psi^{\prime}=\frac{m x_{a}}{m_{C^{\prime}}}$ which is in $I$ since $I$ is $P$-stable, and so $\psi^{\prime} \in \mathcal{F}$. In this way we can successively increase $\psi$ by isotone maps in $\mathcal{F}$ and eventually reach $\tilde{\psi}$. Thus the latter is in $\mathcal{F}$ and so $\mathcal{F}$ is a poset filter.

Example 3.15. The ideal $I=(a, b, c)^{2} \subseteq \mathbb{k}[a, b, c]$ is $P$-stable when $P$ is either the chain or the antichain:

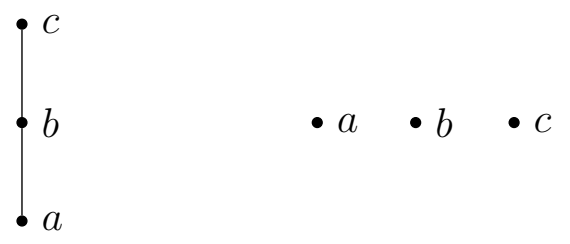

However it is not $P$-stable if $P$ is

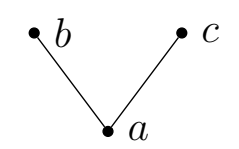

Since $b c \in I$, being $P$-stable would imply $a \in I$, which is not so.

Proposition 3.16. Let $\mathfrak{m}=\left(x_{p}\right)_{p \in P}$ be the maximal ideal of $\mathbb{k}\left[x_{P}\right]$, and $d \geq 2$. Then the power $\mathfrak{m}^{d}$ is $P$-stable iff $P$ is a disjoint union of posets whose Hasse diagrams are rooted trees, with the roots on top.

Proof. Suppose there are elements $a, b_{1}, b_{2}$ of $P$ with $a<b_{1}$ and $a<b_{2}$ and $\left\{b_{1}, b_{2}\right\}$ an antichain. Then $m=b_{1} b_{2}^{d-1} \in \mathfrak{m}^{d}$. The longest $b_{1}$-chain in $m$ is $b_{1}$ which goes through $a$. The longest $b_{2}$-chain is $b_{2} \geq \cdots \geq b_{2}$ a number of $d-1$ times, which also goes through $a$. By Proposition [3.11, $a b_{2}^{d-2} \in \mathfrak{m}^{d}$ which is not so. Thus each $a$ in $P$ has at most one cover and so $P$ is a disjoint union of rooted trees with roots at the top. 
On the other hand let $P$ be a disjoint union of posets whose Hasse diagrams are trees with roots on top. If $B$ is an antichain consisting of elements $\geq a$, then $B=\{b\}$ for a single element $b$. If a longest $b$-chain in a monomial goes through $a$, the criterion for a monomial ideal $I \subseteq \mathbb{k}\left[x_{P}\right]$ being $P$-stable is that if $x_{b} m \in I$ then $x_{a} m \in I$. This holds for $I=\mathfrak{m}^{d}$.

3.3. Primary decomposition. Let $S$ be a subset of $P$. A monomial prime ideal in $\mathbb{k}\left[x_{P}\right]$ is of the form $\mathfrak{p}(S)=\left(x_{p}\right)_{p \in S}$.

Lemma 3.17. Let $I \subseteq P$ be a poset ideal. Then $\mathfrak{p}(I)$ is a P-stable monomial prime ideal. All P-stable monomial prime ideals are of this form.

Proof. If $I \subseteq P$ is a poset ideal, then clearly $\mathfrak{p}(I)$ is $P$-stable. Conversely, let $S \subseteq P$ and suppose $\mathfrak{p}(S)$ is $P$-stable. Let $b \in S$ so $x_{b} \in \mathfrak{p}(S)$. Then for $a<b$, the longest $b$-chain in $x_{b}$ goes through $a$ (it is just $b$ itself), and so $x_{a} \in \mathfrak{p}(S)$. Thus $S$ is a poset ideal.

Proposition 3.18. Let $L \subseteq \mathbb{k}\left[x_{P}\right]$ be a P-stable ideal. If $\mathfrak{p}$ is an associated prime ideal of $L$, then $\mathfrak{p}=\mathfrak{p}(I)$ for some poset ideal $I \subseteq P$.

Proof. Let $\mathfrak{p}(S)$ be a prime ideal annihilating $\bar{m} \in \mathbb{k}\left[x_{P}\right] / L$, so $m x_{b} \in L$ for $b \in S$. Let $a<b$. Then $m x_{a}^{r} x_{b} \in L$. For $r$ large the longest $b$-chain will go through $a$. Therefore $m x_{a}^{r+1} \in L$. If $m x_{a} \notin L$ then $x_{a} \notin \mathfrak{p}(S)$ but $x_{a}^{r+1} \in \mathfrak{p}(S)$. This contradicts $\mathfrak{p}(S)$ being prime.

\section{PRINCIPAL POSET IDEALS}

In this section $\mathcal{J} \subseteq \operatorname{Hom}(P, \mathbb{N})$ will be a finite poset ideal. Then we actually have $\mathcal{J} \subseteq \operatorname{Hom}(P,[n])$ for some $n$ and this is the situation studied in the previous articles [6] and [2].

By Corollary 3.6 the monomial ideal $L^{p}(\mathcal{J}, P) \subseteq \mathbb{k}\left[x_{P}\right]$ is then an artinian monomial ideal, and so a Cohen-Macaulay ideal. Since this quotient ring is obtained by cutting down from $\mathbb{k}\left[x_{\operatorname{Supp}(\mathcal{J})}\right] / L(\mathcal{J}, P)$ by a regular sequence, we see, [6, Thm. 5.9], that $L(\mathcal{J}, P)$ is a Cohen-Macaulay ideal when $\mathcal{J}$ is a finite poset ideal.

Remark 4.1. In [2] it is shown that when $\mathcal{J}$ is a finite poset, the letterplace ideal $L(\mathcal{J}, P)$ defines a simplicial ball (save a few exceptions). Its boundary is therefore a simplicial sphere, whose Stanley-Reisner ideal is also precisely described in [2, Section $5]$

For a finite poset ideal $\mathcal{J}$ of $\operatorname{Hom}(P, \mathbb{N})$ we define the hull map $\alpha(p)=\max \{\phi(p) \mid p \in$ $\mathcal{J}\}$.

Lemma 4.2. When $\mathcal{J} \subseteq P \times \mathbb{N}$ is a finite poset ideal, its hull map $\alpha$ is an isotone map, and

$$
\operatorname{Supp}(\mathcal{J})=\{(p, i) \mid i \leq \alpha(p)\}
$$

This support is a finite poset ideal in $P^{o p} \times \mathbb{N}$.

Proof. This is immediate to verify. 
Remark 4.3. When $\mathcal{J}$ is not a finite poset ideal, the hull map needs not be isotone, and the support $\operatorname{Supp}(\mathcal{J})$ needs not be a poset ideal in $P^{\text {op }} \times \mathbb{N}$.

A class of finite poset ideals now comes out as distinguished: The poset ideals consisting av all isotone maps $\phi$ less than or equal to the hull map.

Definition 4.4. Given an isotone map $\alpha: P \rightarrow \mathbb{N}$ it induces a finite poset ideal in $\operatorname{Hom}(P, \mathbb{N})$ :

$$
\mathcal{J}(\alpha)=\{\psi \mid \psi \leq \alpha\}
$$

The principal co-letterplace ideal $L(P, \alpha)$ is $L(P, \mathcal{J}(\alpha))$ and the principal letterplace ideal $L(\alpha, P)$ is its Alexander dual $L(\mathcal{J}(\alpha), P)$.

Example 4.5. When $P$ is an antichain $\underline{m}=\{1,2, \cdots, m\}$, and $p: \underline{m} \times \mathbb{N} \rightarrow \underline{m}$ the projection to the first coordinate, then $L^{p}(\alpha, P)$ is the complete intersection of monomials $x_{1}^{\alpha(1)+1}, x_{2}^{\alpha(2)+1}, \ldots, x_{m}^{\alpha(m)+1}$. Thus principal letterplace ideals for general $P$ may in some way be considered as generalizations of complete intersections.

Example 4.6. When $\alpha$ is the constant function $\alpha(p)=n+1$, then the principal coletterplace ideal $L(P, \alpha)$ is the co-letterplace ideal $L(P, n+1)$ of [6] (note that we get $n+1$ here since in [6] the chain $[n]$ starts with 1 but here $\mathbb{N}$ starts with 0 , so the chain $[n+1]$ is isomorphic to the chain $[0, n])$, and the Alexander dual $L(\alpha, P)$ is the letterplace ideal $L(n+1, P)$, see Proposition 4.7 below. In [7] the author and A. Nematbakhsh computes the full deformation families of the letterplace ideals $L(2, P)$ when the Hasse diagram of $P$ is a rooted tree. The deformation theory, in analog with complete intersections, is extremely nice.

In the case of a principal poset ideal $\mathcal{J}(\alpha)$ there is a quite nice description of the minimal generators of the letterplace ideal $L(\alpha, P)$.

For an isotone map $\alpha: P \rightarrow \mathbb{N}$ let $[0, i]$ be the integers from 0 to $i$, and define the poset ideal $I_{i}=\alpha^{-1}([0, i])$. We get a filtration of poset ideals of $P$ :

$$
I_{0} \subseteq I_{1} \subseteq \cdots \subseteq I_{n} \subseteq \cdots .
$$

Theorem 4.7. The principal letterplace ideal $L(\alpha, P)$ is generated by the monomials $m_{\Gamma \phi}$ associated to isotone maps $\phi:[0, i] \rightarrow P$ such that $\phi(i) \in I_{i}$. The minimal generators are such $\phi$ with $\phi(j) \notin I_{j}$ for $j<i$.

The notion of principal ideal in a ring is an ideal generated by one element. In contrast, principal letterplace ideals may have many generators.

Example 4.8. Let $\alpha:[3] \rightarrow \mathbb{N}$ be the isotone map

$$
1 \mapsto 1, \quad 2 \mapsto 1, \quad 3 \mapsto 2 .
$$

The variables involved in the hull of $\alpha$ are the variables in

$$
\begin{aligned}
& \left.\left[\begin{array}{lll} 
& & x_{32} \\
x_{11} & x_{21} & x_{31} \\
x_{10} & x_{20} & x_{30}
\end{array}\right], \quad \begin{array}{llll} 
& & & \bullet \\
\bullet & \bullet & \bullet \\
\bullet & \bullet & \bullet
\end{array}\right] . \\
& \begin{array}{lll}
1 & 2 & 3
\end{array}
\end{aligned}
$$


The letterplace ideal $L(\alpha,[3])$ is minimally generated by the following monomials

$$
\begin{array}{ll}
x_{10} x_{11} & x_{10} x_{31} x_{32} \\
x_{10} x_{21}, & x_{20} x_{31} x_{32} \\
x_{20} x_{21} & x_{30} x_{31} x_{32} .
\end{array}
$$

In general for an isotone map $\alpha:[m] \rightarrow \mathbb{N}$ the minimal generators of $L(\alpha,[m])$ are

$$
x_{\phi(0), 0} x_{\phi(1), 1} \cdots x_{\phi(r), r}
$$

with $\phi$ isotone and $x_{\phi(j), j}$ in column $\phi(j)$ and row $j$. The last variable $x_{\phi(r), r}$ is in top of its column i.e. $\alpha \circ \phi(r)=r$, while the previous variables are not in top of their columns, i.e. $\alpha \circ \phi(j)>j$.

Before proving the above theorem we develop some lemmata.

Lemma 4.9. Let $(p, i) \in \Lambda \phi$ where $i \geq 1$. Then there is a $q \leq p$ such that $(q, i-1) \in \Lambda \phi$.

Proof. If every $q<p$ has $\phi(q)<i$ then $(p, i-1) \in \Lambda \phi$. Otherwise there is a minimal $q<p$ with $\phi(q)=i$. Then $(q, i-1) \in \Lambda \phi$.

Lemma 4.10. Suppose $\psi$ is not $\leq \phi$. Then there is $(p, i) \in \Lambda \psi$ such that $i \geq \phi(p)$.

Proof. Let $p$ be minimal such that $\psi(p)>\phi(p)$. Let $i=\psi(p)-1 \geq \phi(p)$. Then if $q<p$

$$
\psi(q) \leq \phi(q) \leq \phi(p)<\psi(p) .
$$

So we see that $(p, i) \in \Lambda \psi$.

Lemma 4.11. Suppose $\phi(p)=r$. For any multichain $p_{0} \leq p_{1} \leq \cdots \leq p_{r}=p$, there is some $\psi$ which is not $\leq \phi$ such that

$$
\Lambda \psi=\left\{\left(p_{0}, 0\right),\left(p_{1}, 1\right), \ldots,\left(p_{r}, r\right)\right\} .
$$

Proof. Let $F_{0}=P$ and for $i=1, \ldots, r+1$ let $F_{i}$ consist of all elements $\geq p_{i-1}$. For $p \in F_{i} \backslash F_{i+1}$ define $\psi(p)=i$. Note that if

$$
p_{u-1}<p_{u}=\ldots=p_{v}<p_{v+1},
$$

then $\psi\left(p_{u}\right)=\cdots=\psi\left(p_{v}\right)=v+1$ and $\psi\left(p_{u-1}\right)=u$. We see that $\psi\left(p_{r}\right)>r$ and so $\psi$ is not $\leq \phi$. We see that all $\left(p_{i}, i\right)$ are in $\Lambda \psi$, and will show that these are precisely the elements of $\Lambda \psi$.

Let $(q, t) \in \Lambda \psi$ with $\psi(q)=i+1$. Then $t \leq i$ and $q \in F_{i+1} \backslash F_{i+2}$ so $q \geq p_{i}$. Then since $F_{i+1} \backslash F_{i+2}$ is nonempty, $p_{i+1}>p_{i}$ and so $\phi\left(p_{i}\right)=i+1$. If $q>p_{i}$ we could not have $(q, t) \in \Lambda \psi$. Thus $q=p_{i}$. Let $p_{j-1}<p_{j}=\cdots=p_{i}<p_{i+1}$. Then $\phi\left(p_{j-1}\right)=j$ and we must have $j \leq t \leq i$. Thus $(q, t)=\left(p_{t}, t\right)$.

Proposition 4.12. Given a principal poset ideal $\mathcal{J}(\phi)$. The inclusion minimal elements of the sets $\Lambda \psi$ for $\psi \in \mathcal{J}(\phi)^{c}$ are sets

$$
\left\{\left(p_{0}, 0\right),\left(p_{1}, 1\right), \ldots,\left(p_{r}, r\right)\right\}
$$

where:

- $p_{0} \leq p_{1} \leq \cdots \leq p_{r}$ is a multichain

- $\phi\left(p_{r}\right)=r$

- $\phi\left(p_{i}\right)>i$ for $i<r$. 
Proof. By Lemma 4.11 every such chain is $\Lambda \psi$ for some $\psi$ not $\leq \phi$. By Lemma 4.10 we cannot omit $\left(p_{r}, r\right)$ from such a chain, and by Lemma 4.9 we cannot omit $\left(p_{i}, i\right)$ for $i<r$.

Proof of Theorem 4.7. This is immediate from Definition 1.9 and the proposition above.

Example 4.13. A class of determinantal ideals considered by Bruns and Vetter in [1] and Herzog and Trung in [11] comes from a pair of integer sequences $M$ :

$$
\begin{aligned}
& 0=a_{0}<a_{1}<a_{2}<\cdots<a_{r}<a_{r+1}=m+1 \\
& 0=b_{0}<b_{1}<b_{2}<\cdots<b_{r}<b_{r+1}=n+1 .
\end{aligned}
$$

The ideals $I_{M}$ is generated by the $t$-minors of the matrix $\left(x_{i j}\right)$ whose indices is the hook subset of $[1, m] \times[1, n]$ consisting of pairs $(a, b)$ where $a<a_{t}$ or $b<b_{t}$, and successively letting $t=1,2, \ldots, r+1$. We explain here how the initial ideal of $I_{M}$ for a diagonal term order is a regular quotient of certain principal letterplace ideals.

Given sequences

$$
\begin{aligned}
& 0=\alpha_{0} \leq \alpha_{1} \leq \alpha_{2} \leq \cdots \leq \alpha_{r} \leq \alpha_{r+1}=m-r \\
& 0=\beta_{0} \leq \beta_{1} \leq \beta_{2} \leq \cdots \leq \beta_{r} \leq \beta_{r+1}=n-r
\end{aligned}
$$

for $i=0, \ldots, r$ define $H_{i}$ to be the hook subset of $\left[\alpha_{i}+1, m-i\right] \times\left[\beta_{i}+1, n-i\right]$ consisting of all elements $(a, b)$ with $a \leq \alpha_{i+1}$ or $b \leq \beta_{i+1}$. (So $H_{0}$ is the whole $[1, m] \times[1, n]$ matrix with the submatrix $\left[\alpha_{1}+1, m\right] \times\left[\beta_{1}+1, n\right]$ removed.) Let $C_{i}=H_{0} \cup H_{1} \cup \cdots \cup H_{i}$ and $C=\cup_{i=0}^{r} H_{i}$ which is a subset of $[1, m] \times[1, n]$ with the induced poset structure. The filtration $C_{0} \subseteq C_{1} \subseteq \cdots$ defines a map $\tau: C \rightarrow \mathbb{N}$. The support of the principal letterplace ideal $L(\tau, C)$ is the following subset of $C \times \mathbb{N}$ :

$$
\tilde{C}=(C \times\{0\}) \cup\left(\left(C \backslash C_{0}\right) \times\{1\}\right) \cup\left(\left(C \backslash C_{1}\right) \times\{2\}\right) \cup \cdots .
$$

This is a finite subset. Let $\phi: \tilde{C} \rightarrow \mathbb{N} \times \mathbb{N}$ be the natural map sending an element $(a, b, p)$ of $\left(C \backslash C_{p-1}\right) \times\{p\}$ to $(a+p, b+p)$. The map $\phi$ is an isotone map with bistrict chain fibers. The image of $\phi$ is in $B=[1, m] \times[1, n]$ and we get the ideal $L^{\phi}(\tau, C) \subseteq \mathbb{k}\left[x_{B}\right]$. For the sequences (5) we let $a_{i}=\alpha_{i}+i$ and $b_{i}=\beta_{i}+i$. Then the initial ideal of $I_{M}$ with respect to a diagonal term order, is the ideal $L^{\phi}(\tau, C)$.

\section{Determinantal ideals SPeCializing to PRINCIPAl LeTterplaCe ideals}

We consider isotone maps $\phi:[m] \rightarrow \mathbb{N}$ where $[m]$ is the chain on $m$ elements. We will find determinantal ideals whose initial ideals are the principal letterplace ideals $L(\phi,[m])$. This is a class of determinantal ideals which seemingly has not been considered before. It is a class of determinantal ideals which naturally generalizes the ideals generated by the maximal minors of a matrix of distinct variables: If $\phi:[m] \rightarrow \mathbb{N}$ is the constant map sending each $i \mapsto n$, then $L(\phi,[m])$ is the initial ideal of the ideal of maximal minors of an $(n+1) \times(m+n)$ matrix of distinct variables.

The naturality of this wider class of ideals is shown by the following analogy: If $\underline{m}$ is the antichain on $m$ elements, and $\psi: \underline{m} \rightarrow \mathbb{N}$ the constant map sending $i \mapsto n$, the letterplace ideal $L(\psi, \underline{m})$ is the complete intersection of $m$ monomials all of the same 
degree $n$. On the other hand if $\psi$ sends $i \mapsto d_{i}$, then the letterplace ideal is the complete intersection of monomials of degrees $d_{1}+1, d_{2}+1, \ldots, d_{m}+1$.

\subsection{A new class of determinantal ideals. Given a sequence}

$$
\ell: \ell_{a} \leq \ell_{a+1} \leq \cdots \leq \ell_{b}
$$

To this sequence we associate a matrix $M(\ell)$ with columns indexed by $\ell_{a}+1, \ell_{a}+2, \ldots, \ell_{b}$ and rows indexed by $a, a+1, \ldots, b-1$. For $p \in\left[\ell_{c}+1, \ell_{c+1}\right]$ we put the variable $y_{p, i}$ in position $(p, i)$ if $a \leq i \leq c$ and we put 0 in position $(p, i)$ if $i>c$. Let $k[Y$; $\ell]$ be the polynomial ring generated by these variables.

For $a<c \leq b$ denote by $J_{a}^{c}$ the ideal generated by the $(c-a)$-minors (maximal minors) of the submatrix of $M(\ell)$ whose columns are $\ell_{a}+1, \ell_{a}+2, \ldots, \ell_{c}$ and rows $a, a+1, \ldots, c-1$. Let

$$
I(\ell)=\sum_{c=a+1}^{b} J_{a}^{c} .
$$

Example 5.1. To the sequence \begin{tabular}{c|c|c|c|c|c}
$l_{0}$ & $l_{1}$ & $l_{2}$ & $l_{3}$ & $l_{4}$ \\
\hline 0 & 0 & 3 & 4 & 6
\end{tabular} there is associated a $4 \times 6$ matrix with variables in the indicated positions

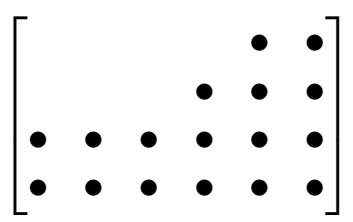

The ideal $I(\ell)$ is the ideal generated by the 2 -minors of the lower left $2 \times 3$ matrix, the 3 -minors of the lower left $3 \times 4$-matrix, and the 4 -minors of the whole matrix.

Example 5.2. Let

$$
\ell: 0=\ell_{0}=\cdots=\ell_{n} \leq \ell_{n+1}=n+m .
$$

Then $M(\ell)$ is an $(n+1) \times(n+m)$-matrix of distinct variables. The ideal $I(\ell)$ is the ideal of maximal minors of $M(\ell)$. It is shown in [6, Subsec.3.3], or originally [14], that the initial ideal of $I(\ell)$ is the letterplace ideal $L(n+1,[m]$ ) (with the variables suitably reindexed). This example is our induction start for the proof of the main Theorem 5.6 of this section

We shall show that $I(\ell)$ is a Cohen-Macaulay ideal of codimension the maximum of $\ell_{d}-\ell_{a}-(d-a)+1$ for $d=a+1, \ldots, b$, and that its initial ideal is a principal letterplace ideal $L(\phi,[m])$. It is worth noting the following.

Lemma 5.3. Let $a<c \leq b$ and suppose $l_{d}-l_{c} \leq d-c$ for $d \geq c$. Then $I\left(\ell_{a}, \ldots, \ell_{b}\right)=$ $I\left(\ell_{a}, \ldots, \ell_{c}\right)$.

Proof. Let $c<d \leq b$ and consider a determinant $D$ of size $d-a$ in $J_{a}^{d}$. It is given by columns in $M(\ell)$ in positions $p_{a+1}, p_{a+2}, \ldots, p_{d}$, and $p_{d} \leq \ell_{d}$. Then by hypothesis $p_{c} \leq \ell_{c}$. If $p_{c+1} \leq \ell_{c}$, then the upper $d-c$ rows has at most $d-c-1$ nonzero columns, and the determinant is zero. If $p_{c+1}>\ell_{c}$ then by the hypothesis we must have $p_{r}=\ell_{r}=\ell_{c}+r-c$, for $r=c+1, \ldots, d$. The upper $d-c$ rows then is only nonzero in the 
last $d-c$ columns, which is a lower triangular matrix with determinant $D_{2}$. Thus the determinant is $D$ is a product of $D_{1} \cdot D_{2}$ where $D_{1}$ is a $(c-a)$-determinant consisting of the first $c-a$ columns and the rows $p_{a+1}, \ldots, p_{c}$.

5.2. Sequences and letterplace ideals. For integers $s \leq t$ let $[s, t]$ be the interval of integers $r$ such that $s \leq r \leq t$. From an isotone map $\phi:[1, m] \rightarrow \mathbb{N}$ we get the letterplace ideal $L(\phi,[m])$. To gain more flexibility we can take an interval $[u+1, v]$ with $m=v-u$ and an integer $a \geq 0$ and identify $\phi$ with a map $[u+1, v] \rightarrow \mathbb{N}_{\geq a}$ and get a letterplace ideal $L(\phi,[u+1, v])$ which we can identify with $L(\phi,[m])$. The isotone map $\phi:[u+1, v] \rightarrow \mathbb{N}_{\geq a}$ may be given by a sequence

$$
i: u=i_{a} \leq i_{a+1} \leq \cdots \leq i_{b}=v
$$

such that $\phi(p)=c$ for $p \in\left[i_{c}+1, i_{c+1}\right]$. Let $\mathbb{k}[X ; i]$ be the polynomial ring with variables $x_{p, i}$ where $a \leq i \leq c$ for $p \in\left[i_{c}+1, i_{c+1}\right]$. We get a principal letterplace ideal $L\left(\phi ;\left[i_{a}+1, i_{b}\right]\right)$ in $\mathbb{k}[X ; i]$, which we denote by $L(i)$.

To the sequence $i$ we associate a sequence $\ell$ given by

$$
\begin{cases}l_{a}=i_{a}+a, & \\ l_{c}=i_{c}+c-1, & i_{c}>i_{c-1} \\ l_{c}=l_{c-1}, & i_{c}=i_{c-1} .\end{cases}
$$

Let $1 \leq c<d \leq b$ be indices such that $l_{d}>l_{d-1}$ and $l_{c}>l_{c-1}$, so $l_{d}$ and $l_{c}$ are the last columns in $M(\ell)$ with precisely $d$, resp. $c$ variables, then

$$
l_{d}-l_{c}=i_{d}-i_{c}+(d-c)>d-c .
$$

A sequence with this property is called a terrace sequence. It means that in the associated matrix $M(\ell)$, for a step the horizontal part is $>$ the vertical part, except possibly for the first step where one has $\geq$. Given a terrace sequence, we may associate a weakly increasing sequence (17) by

$$
\begin{cases}i_{a}=l_{a}-a & \\ i_{c}=l_{c}-c+1, & l_{c}>l_{c-1} \\ i_{c}=i_{c-1}, & l_{c}=l_{c-1} .\end{cases}
$$

This gives a one-to-one correspondence between weakly increasing sequences $i$ and terrace sequences $\ell$. To facilitate our argument later, we need to introduce some more notions on sequences. Given any weakly increasing sequence $\ell$ we can associate a terrace sequence $\ell^{\prime}$ as follows. Consider

$$
l_{a}-a, l_{a+1}-a, l_{a+2}-(a+1), \cdots, l_{b}-(b-1) .
$$

Let the successive maxima after $m_{0}=l_{a}-a$ as we move from left to right be for indices $m_{1}, \ldots, m_{r}$ where by convention the last maximum is $\infty$ in position $m_{r}=b+1$.

Example 5.4. Let

$$
l_{2}=3,3,5,7,8,11=l_{7}
$$


The associated matrix $M(\ell)$ of $y$-variables is (with bullets indicating variables):

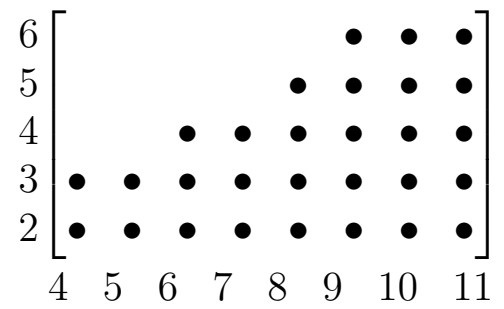

The difference sequence is

$$
l_{2}-2=1,1,2,3,3,5=l_{7}-6 .
$$

The successive maxima after 1 as we move from left to right are 2, 3, 5 and $\infty$ in positions $4,5,7$ and 8 (by the convention).

Let $\ell_{m_{i}}^{\prime}=\ell_{m_{i}}$ and for $p \in\left[m_{i}, \ldots m_{i+1}-1\right]$ let $\ell_{p}^{\prime}=\ell_{m_{i}}$. This is a terrace sequence, actually the largest terrace sequence which is $\leq \ell$. And this terrace sequence corresponds to a weakly increasing sequence $i$. Note that the matrix $M(\ell)$ is a submatrix of $M\left(\ell^{\prime}\right)$.

Example 5.5. Continuing the example above we get

$$
l_{2}^{\prime}=3,3,5,7,7,11=l_{7}^{\prime} .
$$

The associated matrix $M\left(\ell^{\prime}\right)$ of $y$-variables is (with bullets indicating variables):

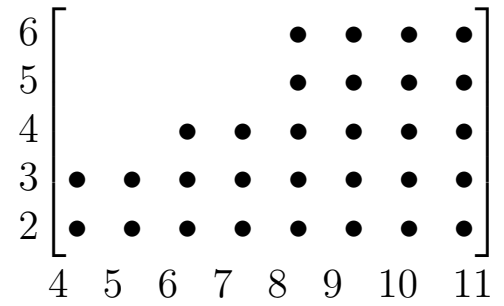

The associated $i$-sequence to $\ell$ and $\ell^{\prime}$ is

$$
i_{2}=1,1,2,3,3,5=i_{7} .
$$

We may display the associated set of $x$-variables as:

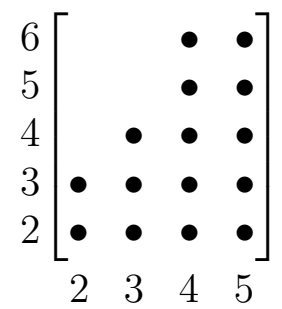

We may think of the columns of the matrix of $x$-variables above as being transported to diagonals in the matrices $M(\ell)$ and $M\left(\ell^{\prime}\right)$, but we see that there will be additional $y$-variables in these matrices which we use for determinants. 
5.3. The main statement. We get a map of polynomial rings $\mathbb{k}[X ; i] \rightarrow \mathbb{k}[Y ; \ell]$ given by $x_{p, i} \mapsto y_{p+i, i}$ so we are shifting the columns of the $x$-matrix to north-east diagonals in the $y$-matrix. The image of the letterplace ideal $L(i)$ then generates an ideal in $\mathbb{k}[Y ; \ell]$ which we denote $L^{Y}(i)$.

Theorem 5.6. Given a weakly increasing sequence $\ell$, let $\ell^{\prime}$ the largest terrace sequence $\leq \ell$, and $i$ its associated weakly increasing sequence. For a diagonal term order on $\mathbb{k}[Y ; \ell]$ we have in $(I(\ell))=L^{Y}(i)$.

Hence $I(\ell)$ is a Cohen-Macaulay ideal of codimension equal to

$$
i_{b}-i_{a}=l_{b}^{\prime}-l_{a}^{\prime}-(b-a)+1=\max \left\{l_{d}-l_{a}-(d-a)+1 \mid d=a+1, \ldots, b\right\} .
$$

Before proving this we need some lemmata. If $i^{\prime}$ is a segment of the sequence $i$, then $\mathbb{k}\left[X ; i^{\prime}\right]$ is a subring of $\mathbb{k}[X ; i]$. The ideal generated by $L\left(i^{\prime}\right)$ in the latter ring is denoted $\hat{L}\left(i^{\prime}\right)$. Similarly if $\ell^{\prime}$ is a segment of $\ell$, then $\mathbb{k}\left[Y ; \ell^{\prime}\right]$ is a subring of $\mathbb{k}[Y, \ell]$. The ideal generated by $I\left(\ell^{\prime}\right)$ in the latter ring, will be denoted by $\hat{I}\left(\ell^{\prime}\right)$.

Lemma 5.7. Given weakly increasing sequences $i$ and $\ell$ as in (7) and (6) $)$. Let $a \leq c \leq$ $b$.
a. $\hat{L}\left(i_{a}, \ldots, i_{c}\right) \subseteq L\left(i_{a}, \ldots, i_{b}\right) \subseteq \hat{L}\left(i_{a}, \ldots, i_{c}\right)+\hat{L}\left(i_{c}, \ldots, i_{b}\right)$
b. $\hat{I}\left(l_{a}, \ldots, l_{c}\right) \subseteq I\left(l_{a}, \ldots, l_{b}\right) \subseteq \hat{I}\left(l_{a}, \ldots, l_{c}\right)+\hat{I}\left(l_{c}, \ldots, l_{b}\right)$

Proof. The first inclusions are immediate from the definitions of these ideals. We then prove the second inclusions.

a. By Proposition 4.7 a minimal generator of $L\left(i_{a}, \ldots, i_{b}\right)$ is the monomial associated to

$$
\left(p_{a}, a\right),\left(p_{a+1}, a+1\right), \ldots,\left(p_{d}, d\right)
$$

where $p_{s}>i_{s+1}$ for $s<d$ (due to minimality of the generator) and $p_{d} \leq i_{d+1}$. If $d<c$, this monomial is in $L\left(i_{a}, \ldots, i_{c}\right)$. If $d \geq c$ then the monomial associated to $\left(p_{c}, c\right), \ldots,\left(p_{d}, d\right)$ is in $L\left(i_{c}, \ldots, i_{b}\right)$.

b. Suppose we have a $(d-a)$-determinant in $J_{a}^{d}$ where $d \leq b$. If $d \leq c$ then clearly this determinant is in the first summand. Suppose $d>c$. Let the columns of the determinant be in positions $q_{a+1}, \ldots, q_{d}$. Since this determinant is in $J_{a}^{d}$ we have $q_{d} \leq l_{d}$. Then by expanding the determinant by the upper $(d-c)$ rows we see that the determinant is in the second summand.

Lemma 5.8. Suppose $l_{c}<l_{c+1}$ and let $i$ be associated to $\ell$. Then the variable $y_{l_{c}+1, c}$ does not occur in a minimal generator of $L^{Y}(i)$.

Proof. If there is a previous maximum $l_{d}+1-d$ in the sequence (8) at or before $l_{c}+1-c$, let $d$ be maximal such. Then $d \leq c$ and and $l_{d}+1-d \geq l_{c}+1-c$. Then $l_{d}^{\prime}=l_{d}$ and $i_{d}=l_{d}+1-d$. But then there is no variable $x_{i_{d}, d}$ and so no variable $x_{l_{c}+1-c, c}$ and so no variable $y_{l_{c}+1, c}$ in $L^{Y}(i)$.

If there is no previous maximum in the sequence (8) before $l_{c}+1-c$, then $l_{a}-a \geq$ $l_{c}+1-c$ and an analog argument implies that there is no $y_{l_{c}+1, c}$ variable. 
Given $\ell$ with $l_{c}<l_{c+1}$. Let

$$
\begin{aligned}
& \ell^{\prime}: l_{a} \leq \cdots \leq l_{c}+1 \leq l_{c+1} \leq \cdots \leq l_{b} . \\
& \ell_{c}: l_{a} \leq \cdots \leq l_{c} \\
& \ell_{c}^{\prime}: l_{a} \leq \cdots \leq l_{c}+1 \\
& \ell^{c}: l_{c}+1 \leq \cdots \leq l_{b}
\end{aligned}
$$

Let $i$ be associated to $\ell$. We then correspondingly have $i^{\prime}, i_{c}, i_{c}^{\prime}, i^{c}$. The $\operatorname{ring} \mathbb{k}\left[Y ; \ell^{\prime}\right]$ is $\mathbb{k}[Y ; \ell] /\left(y_{l_{c}+1, c}\right)$. Let $\bar{I}(\ell)$ be the image of $I(\ell)$ in the latter ring. Also let $\hat{I}\left(\ell_{c}\right)$ be the ideal generated by $I\left(\ell_{c}\right)$ in this ring, and so on.

Lemma 5.9. $\quad$ a. $\bar{I}(\ell) \subseteq \hat{I}\left(\ell_{c}\right)+\hat{I}\left(\ell^{c}\right)$

b. $I\left(\ell^{\prime}\right)=\bar{I}(\ell)+\hat{I}\left(\ell_{c}^{\prime}\right)$

c. $\hat{I}\left(\ell_{c}^{\prime}\right) \cdot \hat{I}\left(\ell^{c}\right) \subseteq \bar{I}(\ell)$

Proof. a. By Lemma 5.7.b we have

$$
I(\ell) \subseteq \hat{I}\left(\ell_{c}\right)+\hat{I}\left(\ell^{c}\right)+\left(y_{l_{c}+1, c}\right)
$$

in $\mathbb{k}[Y ; \ell]$. Then map this down to $\mathbb{k}\left[Y ; \ell^{\prime}\right]$.

b. Given a $(d-a)$-determinant in $J_{a}^{d}$ of $I\left(\ell^{\prime}\right)$ and let $q_{a+1}, \ldots, q_{d}$ be the columns involved in this determinant. If $d \leq c$ then $q_{d} \leq l_{c}+1$ and this determinant is in $\hat{I}\left(\ell_{c}^{\prime}\right)$. If $d>c$ then any such determinant of $I\left(\ell^{\prime}\right)$ and $I(\ell)$ are the same modulo $y_{l_{c}+1, c}$.

c. Let $E$ and $F$ be determinants in the first and second factor. Let $E$ be an $(e-a)$ determinant of $I\left(\ell_{c}^{\prime}\right)$ involving columns $q_{a+1}, \cdots, q_{e}$. So $e \leq c$ and $q_{e} \leq l_{c}+1$. If $q_{e} \leq l_{c}$ then $E$ is in $I(\ell)$ and so in $\bar{I}(\ell)$. Suppose $q_{e}=l_{c}+1$. Then we are considering a $(c-a)$-determinant (by the definition of the ideals in Subsection 5.1), so $e=c$. Let the determinant $F$ have columns in positions $q_{c+1}, \ldots, q_{d}$ where $l_{c}+1<q_{c+1}$. Consider now the determinant with columns $q_{a+1}, \ldots, q_{c}, \ldots, q_{d}$ in $M(\ell)$. We expand it by the upper $(d-c)$ rows. Then this determinant is $E \cdot F$ modulo $y_{l_{c}+1, c}$ and so $E \cdot F \in \bar{I}(\ell)$.

Proof of Theorem 5.6. We are going to use induction on $\ell$. The induction start will be as in Example 5.2, using the sequence

$$
\ell: l_{a}=\cdots=l_{b-1} \leq l_{b}
$$

Then $M(\ell)$ is a $(b-a) \times\left(l_{b}-l_{a}\right)$ matrix $M(\ell)$ of distinct variables, and the statement is classic, [14]. We will successively increase the $l_{i}$ for $i<b$. We then successively replace variables by zero in the matrix $M(\ell)$.

By assumption we have $\operatorname{in}(I(\ell))=L^{Y}(i)$ for some $\ell$ and associated $i$. We want to show that $\operatorname{in}\left(I\left(\ell^{\prime}\right)\right)=L^{Y}\left(i^{\prime}\right)$. Denote by $\bar{L}^{Y}(i)$ the image of $L^{Y}(i)$ in $\mathbb{k}\left[Y ; \ell^{\prime}\right]=$ $\mathbb{k}[Y ; \ell] /\left(y_{\ell_{c}+1, c}\right)$. We note that $L^{Y}\left(i^{\prime}\right)=\bar{L}^{Y}(i)+\hat{L}^{Y}\left(i_{c}^{\prime}\right)$. By Lemma 5.9 b. $I\left(\ell^{\prime}\right)=$ $\bar{I}(\ell)+\hat{I}\left(\ell_{c}^{\prime}\right)$. What we need to show is then

$$
\operatorname{in}\left(\bar{I}(\ell)+\hat{I}\left(\ell_{c}^{\prime}\right)\right)=\bar{L}^{Y}(i)+\hat{L}^{Y}\left(i_{c}^{\prime}\right) .
$$

By induction the following inclusion is clear

$$
\operatorname{in}\left(\bar{I}(\ell)+\hat{I}\left(\ell_{c}^{\prime}\right)\right) \supseteq \bar{L}^{Y}(i)+\hat{L}^{Y}\left(i_{c}^{\prime}\right) .
$$


Consider the exact sequence

$$
0 \leftarrow \frac{\mathbb{k}\left[Y ; \ell^{\prime}\right]}{\hat{I}\left(\ell_{c}^{\prime}\right)+\hat{I}\left(\ell^{c}\right)} \leftarrow \frac{\mathbb{k}\left[Y ; \ell^{\prime}\right]}{\hat{I}\left(\ell_{c}\right)+\hat{I}\left(\ell^{c}\right)} \stackrel{\alpha}{\longleftarrow} \bar{I}(\ell)+\hat{I}\left(\ell_{c}^{\prime}\right) .
$$

We show that the kernel of $\alpha$ is $\bar{I}(\ell)$. That the latter is in the kernel follows by Lemma 5.9. a. Let then $f_{c}^{\prime} \in \hat{I}\left(\ell_{c}^{\prime}\right)$ be in the kernel of $\alpha$, so $f_{c}^{\prime}=f_{c}+f^{c}$ where $f_{c} \in \hat{I}\left(\ell_{c}\right)$ (and so is in $\bar{I}(\ell)$ by Lemma 5.7b.), and $f^{c} \in \hat{I}\left(\ell^{c}\right)$. Then $f_{c}^{\prime}-f_{c}=f^{c}$. But the left side is in the ideal in $\mathbb{k}\left[Y ; \ell^{\prime}\right]$ generated by $I\left(\ell_{c}^{\prime}\right) \subseteq \mathbb{k}\left[Y ; \ell_{c}^{\prime}\right]$, and $f^{c}$ is in the ideal generated by $I\left(\ell^{c}\right) \subseteq \mathbb{k}\left[Y ; \ell^{c}\right]$. These polynomial rings have distinct variables. Then

$$
\hat{I}\left(\ell_{c}^{\prime}\right) \cap \hat{I}\left(\ell^{c}\right)=\hat{I}\left(\ell_{c}^{\prime}\right) \cdot \hat{I}\left(\ell^{c}\right) .
$$

Thus

Hence $f_{c}^{\prime} \in \bar{I}(\ell)$.

$$
f_{c}^{\prime}-f_{c} \in \hat{I}\left(\ell_{c}^{\prime}\right) \cdot \hat{I}\left(\ell^{c}\right) \subseteq \bar{I}(\ell) \quad \text { by Lemma }[5.9 .
$$

There is also an exact sequence

$$
0 \leftarrow \frac{\mathbb{k}\left[Y ; \ell^{\prime}\right]}{\hat{L}^{Y}\left(i_{c}^{\prime}\right)+\hat{L}^{Y}\left(i^{c}\right)} \leftarrow \frac{\mathbb{k}\left[Y ; \ell^{\prime}\right]}{\hat{L}^{Y}\left(i_{c}\right)+\hat{L}^{Y}\left(i^{c}\right)} \leftarrow \bar{L}^{Y}(i)+\hat{L}^{Y}\left(i_{c}^{\prime}\right) \leftarrow \bar{L}^{Y}(i) \leftarrow 0 .
$$

We now compare the Hilbert functions of the terms in the sequences (10) and (11). 1. By induction $L^{Y}\left(i_{c}\right)$ is the initial ideal of $I\left(\ell_{c}\right)$ and so these ideals have the same Hilbert functions. The same also holds true for the pairs $i_{c}^{\prime}, \ell_{c}^{\prime}$, for $i^{c}, \ell^{c}$ and for $i, \ell$.

2. Since $L^{Y}\left(i_{c}^{\prime}\right)$ and $L^{Y}\left(i^{c}\right)$ involve distinct sets of variables, the Hilbert functions of $\hat{L}^{Y}\left(i_{c}^{\prime}\right)+\hat{L}^{Y}\left(i^{c}\right)$ and $\hat{I}\left(\ell_{c}^{\prime}\right)+\hat{I}\left(\ell^{c}\right)$ are the same, and similarly with $i_{c}^{\prime}, \ell_{c}^{\prime}$ replaced by $i_{c}, \ell_{c}$.

3. By Lemma $5.8\left(L^{Y}(i): y_{\ell_{c}+1, c}\right)=L^{Y}(i)$. Since $\operatorname{in}(I(\ell))=L^{Y}(i)$ it is an easy fact that $\left(I(\ell): y_{\ell_{c}+1, c}\right)=I(\ell)$. Hence the Hilbert functions of $\bar{L}^{Y}(i)$ and $\bar{I}(\ell)$ in $\mathbb{k}\left[Y ; \ell^{\prime}\right]=\mathbb{k}[Y ; \ell] /\left(y_{\ell_{c}+1, c}\right)$ are the same.

4. Comparing the sequences (10) and (11)

$$
\bar{L}^{Y}(i)+\hat{L}^{Y}\left(i_{c}^{\prime}\right), \quad I\left(\ell^{\prime}\right)=\bar{I}(\ell)+\hat{I}\left(\ell_{c}^{\prime}\right)
$$

have the same Hilbert function. Taking the inclusion (9) into account, this inclusion must be an equality.

\section{Strongly Stable IDEALS}

We now assume $P$ is the totally ordered poset

$$
P=[m]=\{1<2<\cdots<m\} .
$$

Let $\mathcal{J} \subseteq \operatorname{Hom}([m], \mathbb{N})$ be a poset ideal. We shall show that the associated letterplace ideal gives by projection a strongly stable ideal in $\mathbb{k}\left[x_{[m]}\right]$, and the co-letterplace ideal gives by projection a strongly stable ideal in $\mathbb{k}\left[x_{\mathbb{N}}\right]=\mathbb{k}\left[x_{0}, x_{1}, x_{2}, \ldots\right]$ generated in degree $\leq m$. Each of these correspondences are one-to-one and putting them together we get a duality between strongly stable ideals in $\mathbb{k}\left[x_{[m]}\right]$ and finitely generated $m$-regular strongly stable ideals in $\mathbb{k}\left[x_{\mathbb{N}}\right]$. The results in this section are joint with Alessio D'Ali and Amin Nematbakhsh. 
If $S=\sum_{p \in P} s_{p} p$ we shall often by abuse of notation write $S$ as a short for the monomial $\prod_{p \in P} x_{p}^{s_{p}}$.

6.1. Strongly stable ideals from letterplace ideals. Recall that an ideal $I$ of $\mathbb{k}\left[x_{1}, \ldots, x_{n}\right]$ is strongly stable if $x_{j} m \in I$ and $i<j$ implies $x_{i} m \in I$.

The letterplace ideal $L(\mathcal{J}, P)$ is an ideal in $\mathbb{k}\left[x_{[m] \times \mathbb{N}}\right]$. The projection onto the first factor $[m] \times \mathbb{N} \stackrel{p_{1}}{\longrightarrow}[m]$ has right strict chain fibers, and so we get the projected ideal $L^{p_{1}}(\mathcal{J}, P) \subseteq \mathbb{k}\left[x_{[m]}\right]$ such that $\mathbb{k}\left[x_{[m]}\right] / L^{p_{1}}(\mathcal{J}, P)$ is a regular quotient of $\mathbb{k}\left[x_{S}\right] / L(\mathcal{J}, P)$ for suitable finite $S$.

Theorem 6.1. The projected letterplace ideal $L^{p_{1}}(\mathcal{J},[m])$ is a strongly stable ideal in $\mathbb{k}\left[x_{[m]}\right]$. This correspondence is a one-one correspondence between poset ideals in $\operatorname{Hom}([m], \mathbb{N})$ and strongly stable ideals $\mathcal{J}$ in $\mathbb{k}\left[x_{[m]}\right]$.

Proof. The monomials in $L^{p_{1}}(\mathcal{J},[m])$ are the images $\bar{\Lambda} \phi$ where $\phi \in \mathcal{J}^{c}$. For a map $\phi:[m] \rightarrow \mathbb{N}$ then $\Lambda \phi=\{(a, i) \mid \phi(a)>i \geq \phi(a-1)\}$. This gives $\bar{\Lambda} \phi=\prod_{a=1}^{m} x_{a}^{\phi(a)-\phi(a-1)}$. Suppose that $a \geq 1$ and $\phi(a)>\phi(a-1)$ so $x_{a}$ is a factor of $\bar{\Lambda} \phi$. Define

$$
\phi^{\prime}(i)=\left\{\begin{array}{ll}
\phi(i), & i \neq a-1 \\
\phi(a-1)+1, & i=a-1
\end{array} .\right.
$$

Then $\bar{\Lambda} \phi^{\prime}=\frac{x_{a-1}}{x_{a}} \bar{\Lambda} \phi$ and $\phi^{\prime} \geq \phi$ and so $\phi^{\prime} \in \mathcal{J}^{c}$. This implies that $\bar{\Lambda} \mathcal{J}^{c}=L^{p_{1}}(\mathcal{J}, P)$ is strongly stable.

Since

$$
\operatorname{Hom}([m], \mathbb{N}) \stackrel{\bar{\Lambda}}{\longrightarrow} \text { monomials in } \mathbb{k}\left[x_{1}, \ldots, x_{m}\right]
$$

is a bijection, this gives an injective map from poset ideals $\mathcal{J}$ to strongly stable ideals in $\mathbb{k}\left[x_{1}, \ldots, x_{m}\right]$.

Consider now a strongly stable ideal $I \subseteq \mathbb{k}\left[x_{1}, \ldots, x_{m}\right]$. The monomials in $I$ correspond by Proposition 3.3 via the inverse of $\bar{\Lambda}$ to a subset $\mathcal{F} \subseteq \operatorname{Hom}([m], \mathbb{N})$. We show that $\mathcal{F}$ is a poset filter. Let $\phi \in \mathcal{F}$ and $\psi>\phi$. For some $a$ we must then have $\phi(a)<\psi(a)$. Let $a$ be maximal such. Define

$$
\phi^{\prime}(i)= \begin{cases}\phi(i), & i \neq a \\ \phi(a)+1, & i=a .\end{cases}
$$

Then $\psi \geq \phi^{\prime}>\phi$. But $\bar{\Lambda} \phi=\prod_{a=1}^{m} x_{a}^{\phi(a)-\phi(a-1)}$ and $\bar{\Lambda} \phi^{\prime}=\frac{x_{a}}{x_{a+1}} \cdot \bar{\Lambda} \phi$ if $a<m$ and $\bar{\Lambda} \phi^{\prime}=x_{a} \bar{\Lambda} \phi$ if $a=m$. Then $\bar{\Lambda} \phi^{\prime} \in I$ and so $\phi^{\prime} \in \mathcal{F}$. Continuing we eventually get $\psi \in \mathcal{F}$. Thus $\mathcal{F}$ is poset filter and so $\mathcal{F}=\mathcal{J}^{c}$ for a poset ideal $\mathcal{J}$.

6.2. Strongly stable ideals from co-letterplace ideals. Given the poset ideal $\mathcal{J} \subseteq$ $\operatorname{Hom}([m], \mathbb{N})$ we get the co-letterplace ideal $L([m], \mathcal{J})$. The projection $[m] \times \mathbb{N} \stackrel{p_{2}}{\longrightarrow}$ $\mathbb{N}$ has left strict chain fibers. We then get the ideal $L^{p_{2}}([m], \mathcal{J})$ in $\mathbb{k}\left[x_{\mathbb{N}}\right]$ such that $\mathbb{k}\left[x_{\mathbb{N}}\right] / L^{p_{2}}([m], \mathcal{J})$ is a regular quotient of $\mathbb{k}\left[x_{[m] \times \mathbb{N}}\right] / L([m], \mathcal{J})$.

Theorem 6.2. The projected co-letterplace ideal $L^{p_{2}}([m], \mathcal{J})$ is a strongly stable $m$ regular ideal in $\mathbb{k}\left[x_{\mathbb{N}}\right]$. This gives a one-one correspondence between poset ideals $\mathcal{J} \subseteq$ $\operatorname{Hom}([m], \mathbb{N})$ and finitely generated strongly stable $m$-regular ideals in $\mathbb{k}\left[x_{\mathbb{N}}\right]$. 
Proof. Let $I=[1, i] \subseteq[m]$ be a poset ideal and $\phi:[1, i] \rightarrow \mathbb{N}$ be a marker for $\mathcal{J}$. Then $\Gamma \phi=\{(a, j) \mid \phi(a)=j, 1 \leq a \leq i\}$ and $\overline{\Gamma \phi}$ in $\mathbb{k}\left[x_{\mathbb{N}}\right]$ is $\prod_{a=1}^{i} x_{\phi(a)}$. Take a variable $x_{j}$ with $j \geq 1$ and dividing $\overline{\Gamma \phi}$. Let $a$ be minimal with $\phi(a)=j$. Define

$$
\phi^{\prime}(i)= \begin{cases}\phi(i), & i \neq a \\ \phi(a)-1=j-1, & i=a .\end{cases}
$$

Then $\phi^{\prime}<\phi$ and so $\phi^{\prime} \in \mathcal{J}$ and $\overline{\Gamma \phi^{\prime}}=\frac{x_{j-1}}{x_{j}} \cdot \overline{\Gamma \phi} \in L^{p_{2}}([m], \mathcal{J})$. Then $L^{p_{2}}([m], \mathcal{J})$ is strongly stable.

Conversely, given a finitely generated $m$-regular strongly stable ideal $I \subseteq \mathbb{k}\left[x_{\mathbb{N}}\right]$. Each generator $x_{i_{1}} x_{i_{2}} \cdots x_{i_{r}}$ where $i_{1} \leq i_{2} \leq \cdots \leq i_{r}$ will have $r \leq m$ and gives an isotone map $\alpha:[1, r] \rightarrow \mathbb{N}$. Let $\mathcal{J}$ be the set of isotone map $\phi:[m] \rightarrow \mathbb{N}$ extending such $\alpha$ 's. We claim that $\mathcal{J}$ is a poset ideal.

For this it is sufficient to show that if $\beta \leq \alpha$ then $\overline{\Gamma \beta}$ is in $I$. But $\overline{\Gamma \beta}=x_{j_{1}} x_{j_{2}} \cdots x_{j_{r}}$ where each $j_{k}=\beta(k) \leq \alpha(k)=i_{k}$. Then by strong stability of $I$ we see that $\overline{\Gamma \beta}$ is in $I$ and so $\beta$ is in $\mathcal{J}$.

Corollary 6.3. There are one-to-one correspondences:

Strongly stable ideals in $\mathbb{k}\left[x_{[m]}\right]$

$\stackrel{1-1}{\longleftrightarrow}$ Poset ideals $\mathcal{J} \subseteq \operatorname{Hom}([\mathrm{m}], \mathbb{N})$

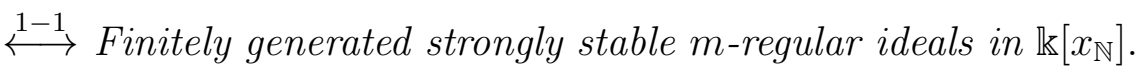

Example 6.4. Let $m=1$. The strongly stable ideal $\left(x_{1}^{n}\right) \subseteq \mathbb{k}\left[x_{1}\right]$ corresponds to the 1-regular ideal $\left(x_{0}, x_{1}, x_{2}, \ldots, x_{n-1}\right) \subseteq \mathbb{k}\left[x_{\mathbb{N}}\right]$.

Let $m=2$. The strongly stable ideal

$$
\left(x_{1}^{a}, x_{1}^{a-1} x_{2}^{b_{1}+1}, \ldots, x_{1}^{a-r} x_{2}^{b_{r}+r}, \ldots, x_{2}^{b_{a}+a}\right)
$$

with $0 \leq b_{1} \leq b_{2} \leq \cdots \leq b_{a}$ corresponds to the smallest 2-regular strongly stable ideal containing

$$
x_{0} x_{b_{a}+a-1}, x_{1} x_{b_{a-1}+a-1}, \cdots, x_{a-1} x_{b_{1}+a-1} .
$$

In the following let $[n]_{0}=\{0<1<\cdots<n\}$ be the chain with $n+1$ elements. By the adjunction between $P \times-$ and $\operatorname{Hom}(P,-)$ in the category of posets, we have

$$
\begin{aligned}
\operatorname{Hom}\left([m],[n]_{0}\right)=\operatorname{Hom}\left([m], \operatorname{Hom}\left([n],[1]_{0}\right)\right) & \left.=\operatorname{Hom}\left([m] \times[n],[1]_{0}\right)\right) \\
& =\operatorname{Hom}\left([n], \operatorname{Hom}\left([m],[1]_{0}\right)\right. \\
& =\operatorname{Hom}\left([n],[m]_{0}\right) .
\end{aligned}
$$

This is the correspondence between a partition of $m$ parts into sizes $\leq n$, and its dual partition of $n$ parts into sizes $\leq m$. We then get the following.

Corollary 6.5. There are one-to-one correspondences:

Strongly stable $n$-regular ideals in $\mathbb{k}\left[x_{[m]}\right]$

$\stackrel{1-1}{\longleftrightarrow}$ Poset ideals $\mathcal{J} \subseteq \operatorname{Hom}\left([m],[n]_{0}\right)$

$\stackrel{1-1}{\longleftrightarrow}$ Strongly stable $m$-regular ideals in $\mathbb{k}\left[x_{[n]}\right]$. 


\section{Proof that the Poset maps induCe REgular SEQuences}

The following is a refinement of Lemma 7.1 of [6], but the proof is exactly the same, and we omit it.

Lemma 7.1. Let $I \subseteq k\left[x_{0}, \ldots, x_{n}\right]$ be a monomial ideal such that every minimal generator of $I$ is squarefree in the variable $x_{0}$, so no minimal generator is divisible by $x_{0}^{2}$. If $f \in S$ is such that $x_{0} f=x_{1} f$ in $\mathbb{k}\left[x_{0}, \ldots, x_{n}\right] / I$, then for every monomial $m$ in $f$ we have $x_{1} m=0=x_{0} m$ in this quotient ring.

Proof of Theorem 2.1. Recall by Lemma 3.2 that an isotone map $\phi: P \rightarrow \mathbb{N}$ corresponds to a chain of poset filters in $P$ :

$$
P=F_{0} \supseteq F_{1} \supseteq \cdots \supseteq F_{n} \supseteq F_{n+1}=\emptyset,
$$

where $\phi(p)=i$ if $p \in F_{i} \backslash F_{i+1}$. Then $\Lambda \phi$ is the set of all pairs $(a, i-1)$ where $a$ is a minimal element in $F_{i}$ for $i \geq 1$.

Let $\phi^{-1}(r)$ have cardinality $\geq 2$. Let $(p, i)$ be the element in the fiber with minimal $i$ and so $\phi^{-1}(r)=R_{1} \cup R_{2}=\{(p, i)\} \cup R_{2}$ is a disjoint union. By Lemma 8.1 in [6] we have a factorization into isotone maps of posets

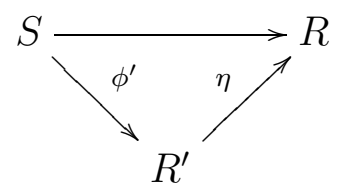

where the cardinality of $R^{\prime}$ is one more than that of $R, \eta^{-1}(r)=\left\{r_{1}, r_{2}\right\}$, and $\phi^{\prime-1}\left(r_{1}\right)=$ $\{(p, i)\}$ and $\phi^{\prime-1}\left(r_{2}\right)=R_{2}$.

For $(p, i) \in S$ denote by $\overline{p, i}$ its image in $R^{\prime}$ and by $\overline{\overline{p, i}}$ its image in $R$. Note that all minimal generators of $L^{\phi^{\prime}}(\mathcal{J}, P)$ are squarefree with respect to $x_{r_{1}}=x_{\overline{p, i}}$. We need to show that $x_{r_{1}}-x_{r_{2}}$ is a non-zero divisor, so $x_{r_{1}} f=x_{r_{2}} f$ implies $f=0$. By Lemma 7.1]it is enough to show that for any monomial $m$, then $x_{r_{1}} m=0=x_{r_{2}} m$ in $\mathbb{k}\left[x_{R^{\prime}}\right] / L^{\phi^{\prime}}(\mathcal{J}, P)$ implies $m=0$ in this quotient ring.

Note. In [6, Theorem 2.1] the proof given in Section 8 there had a minor gap, in that Lemma 7.1 in [6] was not quite sufficient to conclude as above that $m=0$. However Lemma 7.1 above rectifies this.

So assume $m$ is nonzero. We will derive a contradiction. Let $(p, i)$ map to $r_{1}$ and $(q, j)$ map to $r_{2}$. For $\phi$ inclusion minimal in the complement $\mathcal{J}^{c}$, the ascent $\Lambda \phi$ lives in $\mathbb{N} S$, and denote by $\overline{\Lambda \phi}$ its image in the monoid $\mathbb{N} R^{\prime}$. There are then $\phi, \psi \in \mathcal{J}^{c}$ such that $m_{\overline{\Lambda \phi}}$ divides $x_{\overline{p, i}} m$ and $m_{\overline{\Lambda \psi}}$ divides $x_{\overline{q, j}} m$. Let $\phi$ and $\psi$ correspond to respectively

$$
P=F_{0} \supseteq F_{1} \supseteq \cdots, \quad P=G_{0} \supseteq G_{1} \supseteq \cdots .
$$

Then there is $\left(a_{s}, s-1\right)$ with $a_{s} \in \min F_{s}$ such that $\overline{\left(a_{s}, s-1\right)}=\overline{(p, i)}$, and $\left(b_{t}, t-1\right)$ with $b_{t} \in \min G_{t}$ and $\overline{\left(b_{t}, t-1\right)}=\overline{(q, j)}$.

Since $\overline{\overline{a_{s}, s-1}}=\overline{\overline{b_{t}, t-1}}$ and $\phi$ has right strict chain fibers, we have, say $s<t$ and $a_{s} \geq b_{t}$.

Let $A_{s}$ be the minimal elements of $F_{s}$ and let $F_{s}^{\prime}$ be the filter generated by $A_{s} \backslash\left\{a_{s}\right\}$. We claim that $F_{s}^{\prime} \cup G_{s}=F_{s} \cup G_{s}$. Let $p \in F_{s}$. If $p \geq$ some element in $A_{s} \backslash\left\{a_{s}\right\}$ then 
clearly $p \in F_{s}^{\prime}$. If $p \geq a_{s}$ then $p \geq b_{t}$. But since $t>s$ then $p \in G_{s}$. Now consider the following sequence of poset filters:

$$
P=F_{0} \cup G_{0} \supseteq F_{1} \cup G_{1} \supseteq \cdots \supseteq F_{s-1} \cup G_{s-1} \supseteq F_{s}^{\prime} \cup G_{s} \supseteq F_{s+1} \supseteq \cdots .
$$

This chain corresponds to an isotone map $\phi^{\prime}$ where $\phi^{\prime} \geq \phi$ (since for each $p$ the $p$ 'th term above contains $F_{p}$ ), and so $\phi^{\prime} \in \mathcal{J}^{c}$.

Now for two poset ideals $F, G \subseteq P$, we have $\min (F \cup G) \subseteq(\min F) \cup(\min G)$. Thus for $i \in \mathbb{N}$ the product $\prod_{p \in \min (F \cup G)} x_{(p, i-1)}$ divides the least common multiple of $\prod_{p \in \min F} x_{(p, i-1)}$ and $\prod_{p \in \min G} x_{(p, i-1)}$. But all the variables occurring in each of these monomials will by $S \stackrel{\phi^{\prime}}{\longrightarrow} R^{\prime}$ map to distinct variables, since $\phi$ has right strict chain fibers. Thus we will also have that the product $\prod_{p \in \min (F \cup G)} x_{\overline{p, i-1}}$ divides the least common multiple of $\prod_{p \in \min F} x_{\overline{p, i-1}}$ and $\prod_{p \in \min G} x_{\overline{p, i-1}}$. But then $m_{\overline{\Lambda \phi^{\prime}}}$ constructed from the chain (13), see Lemma [3.2, divides the least common multiple of $m \overline{\Lambda \phi} / x_{\overline{a_{s}, s-1}}$ and $m \overline{\Lambda \psi} / x_{\overline{b_{t}, t-1}}$ which both divide $m$. Hence $m_{\overline{\Lambda \phi^{\prime}}}$ divides $m$, contradicting that $m$ is nonzero in $\mathbb{k}\left[x_{R^{\prime}}\right] / L^{\phi^{\prime}}(\mathcal{J}, P)$.

Proof of Theorem 2.2. By induction on the cardinality of im $\phi$. We may assume we have a factorization

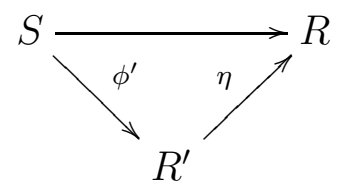

analogous to (12), with $\phi^{-1}(r)$ of cardinality $\geq 2$ and with $\eta^{-1}(r)=\left\{r_{1}, r_{2}\right\}$ and $\phi^{\prime-1}\left(r_{1}\right)=R_{1}=\left\{\left(p_{0}, a\right)\right\}$ and $\phi^{\prime-1}\left(r_{2}\right)=R_{2}$. Furthermore we have by induction established that

$$
\mathbb{k}\left[x_{R^{\prime}}\right] / L^{\phi^{\prime}}(P, \mathcal{J})
$$

is obtained by cutting down from $\mathbb{k}\left[x_{S}\right] / L(P, \mathcal{J})$ by a regular sequence of variable differences.

Let $\left(p_{0}, a\right)$ in $S$ map to $r_{1} \in R^{\prime}$ and $\left(q_{0}, b\right)$ map to $r_{2} \in R^{\prime}$. We will show that $x_{r_{1}}-x_{r_{2}}$ is a regular element in the quotient ring (15). So let $f$ be a polynomial of this quotient ring such that $f\left(x_{r_{1}}-x_{r_{2}}\right)=0$. Then by Lemma 7.1, for any monomial $m$ in $f$ we have $m x_{r_{1}}=0=m x_{r_{2}}$ in the quotient ring $\mathbb{k}\left[x_{R^{\prime}}\right] / L^{\phi^{\prime}}(P, \mathcal{J})$. We assume $m$ is nonzero in this quotient ring and shall derive a contradiction.

There is a minimal marker $i: I \rightarrow \mathbb{N}$ for $\mathcal{J} \subseteq \operatorname{Hom}(P, \mathbb{N})$ such that the monomial $m^{i}=\prod_{p \in I} x_{\overline{p, i_{p}}}$ in $L^{\phi^{\prime}}(P, \mathcal{J})$ divides $m x_{\overline{p_{0}, a}}$, and similarly a minimal marker $j: J \rightarrow \mathbb{N}$ such that the monomial $m^{j}=\prod_{p \in J} x_{\overline{p, j_{p}}}$ divides $m x_{\overline{q_{0}, b}}$. Hence there are $s$ and $t$ in $P$ such that $\overline{s, i_{s}}=\overline{p_{0}, a}$ and $\overline{t, j_{t}}=\overline{q_{0}, b}$. In $R$ we then get:

$$
\overline{\overline{s, i_{s}}}=\overline{\overline{p_{0}, a}}=\overline{\overline{q_{0}, b}}=\overline{\overline{t, j_{t}}}
$$

so $s=t$ would imply $i_{t}=j_{t}$ since $\phi$ has left strict chain fibers. But then

$$
r_{1}=\overline{p_{0}, a}=\overline{s, i_{s}}=\overline{t, j_{t}}=\overline{q_{0}, b}=r_{2}
$$


which is not so. Assume then, say $s<t$. Then $i_{s} \geq j_{t}$ since $\phi$ has left strict chain fibers, and so

$$
i_{t} \geq i_{s} \geq j_{t} \geq j_{s}
$$

In the following let $i_{p}=\infty$ if $p \notin I$ and similarly $j_{p}=\infty$ if $p \notin J$. Form the monomials

- $m_{>s}^{i}=\prod_{p \in I, p>s} x_{\overline{p, i_{p}}}$.

- $m_{i>j}^{i}=\prod_{p \in I, i_{p}>j_{p}, n o t} x_{\overline{p, i_{p}}}$.

- $m_{i<j}^{i}=\prod_{p \in I, i_{p}<j_{p}, n o t(p>s)} x \overline{p, i_{p}}$.

- $m_{i=j}^{i}=\prod_{p \in I, i_{p}=j_{p}, n o t} x_{\overline{p, i_{p}}}$.

Similarly we define $m_{*}^{j}$ for the various subscripts $*$. Then

$$
m^{i}=m_{i=j}^{i} \cdot m_{i>j}^{i} \cdot m_{i<j}^{i} \cdot m_{>s}^{i}
$$

divides $x \overline{s, i_{s}} m$, and

$$
m^{j}=m_{i=j}^{j} \cdot m_{i>j}^{j} \cdot m_{i<j}^{j} \cdot m_{>s}^{j}
$$

divides $x_{\overline{t, j_{t}}} m$.

Now let

$$
\tilde{m}_{i>j}^{j}=\prod_{p \in I \cap J, i_{p}>j_{p}, n o t} x_{\overline{p, j_{p}}},
$$

which is the factor of $m_{i>j}^{j}$ where we take the product only over $I \cap J$ and not over $J$.

There is now a map $\ell: I \rightarrow \mathbb{N}$ defined by

$$
\ell(p)=\left\{\begin{array}{l}
i_{p} \text { for } p \in I, p>s \\
\min \left(i_{p}, j_{p}\right) \text { for } p \in I \text { and not }(p>s) \\
\left(\text { recall that } j_{p}=\infty \text { if } p \notin J\right)
\end{array} .\right.
$$

This is an isotone map as is easily checked. Its associated monomial is

$$
m^{\ell}=m_{i=j} \cdot \tilde{m}_{i>j}^{j} \cdot m_{i<j}^{i} \cdot m_{>s}^{i} .
$$

We will show that this divides $m$. Since the marker $\ell$ is $\leq$ the marker $i$, this will prove the theorem.

Claim 1. $\tilde{m}_{i>j}^{j}$ is relatively prime to $m_{i<j}^{i}$ and $m_{>s}^{i}$.

Proof. Let $x_{p, j_{p}}$ be in $\tilde{m}_{i>j}^{j}$.

1. Suppose it equals the variable $x_{\overline{q, i_{q}}}$ in $m_{i<j}^{i}$. Then $p$ and $q$ are comparable since $\phi$ has left strict chain fibers. If $p<q$ then $j_{p} \geq i_{q} \geq i_{p}$, contradicting $i_{p}>j_{p}$. If $q<p$ then $i_{q} \geq j_{p} \geq j_{q}$ contradicting $i_{q}<j_{q}$.

2 Suppose $x_{\overline{p, j_{p}}}$ equals $x_{\overline{q, i_{q}}}$ in $m_{>s}^{i}$. Then $p$ and $q$ are comparable and so $p<q$ since $q>s$ and we do not have $p>s$. Then $j_{p} \geq i_{q} \geq i_{p}$ contradicting $i_{p}>j_{p}$.

Claim 2. $m_{\ell}$ divides $m x_{\overline{s, i_{s}}}$. 
Proof. Let $a b c=m_{i=j}^{i} \cdot m_{i<j}^{i} \cdot m_{>s}^{i}$ which divides $m x_{\overline{s, i_{s}}}$ and $a b^{\prime}=m_{i=j}^{j} \cdot \tilde{m}_{i>j}^{j}$ which divides $m$ since $x_{\overline{t, j_{t}}}$ is a factor of $m_{>s}^{j}$ since $t>s$. Now if the product of monomials $a b c$ divides the monomial $n$ and $a b^{\prime}$ also divides $n$, and $b^{\prime}$ is relatively prime to $b c$, then the least common multiple $a b b^{\prime} c$ divides $n$. We thus see that the monomial associated to the isotone map $\ell$

divides $m x \overline{s, i_{s}}$.

$$
m^{\ell}=m_{i=j} \cdot \tilde{m}_{i>j}^{j} \cdot m_{i<j}^{i} \cdot m_{>s}^{i}
$$

We need now only show that the variable $x \overline{s, i_{s}}$ occurs to a power in the above product (17) for $m^{\ell}$ less than or equal to that of its power in $m$.

Claim 3. $x_{\overline{s, i_{s}}}$ is not a factor of $\tilde{m}_{i>j}^{j}$ or $m_{i<j}^{i}$.

Proof. 1. Suppose $\overline{s, i_{s}}=\overline{p, i_{p}}$ where $i_{p}<j_{p}$ and not $p>s$. Since $p$ and $s$ are comparable (they are both in a fiber of $\phi$ ), we have $p \leq s$. Since $\phi$ is isotone $i_{p} \leq i_{s}$ and since $\phi$ has left strict chain fibers $i_{p} \geq i_{s}$. Hence $i_{p}=i_{s}$. By (16) $j_{s} \leq i_{s}$ and so $j_{p} \leq j_{s} \leq i_{s}=i_{p}$. This contradicts $i_{p}<j_{p}$.

2. Suppose $\overline{s, i_{s}}=\overline{p, j_{p}}$ where $j_{p}<i_{p}$ and not $p>s$. Then again $p \leq s$ and $i_{p} \leq i_{s} \leq j_{p}$, giving a contradiction.

If now $i_{s}>j_{s}$ then $x \overline{s, i_{s}}$ is a factor in $m_{i>j}^{i}$ but by the above, not in $\tilde{m}_{i>j}^{j}$. Since $m^{\ell}$ is obtained from $m^{i}$ by replacing $m_{i>j}^{i}$ with $\tilde{m}_{i>j}^{j}$, we see that $m^{\ell}$ contains a lower power of $x \overline{s, i_{s}}$ than $m^{i}$ and so $m^{\ell}$ divides $m$.

Claim 4. Suppose $i_{s}=j_{s}$. Then the power of $x_{\overline{s, i_{s}}}$ in $m_{>s}^{i}$ is less than or equal to its power in $m_{>s}^{j}$.

Proof. Suppose $\overline{s, i_{s}}=\overline{p, i_{p}}$ where $p>s$. We will show that then $i_{p}=j_{p}$. This will prove the claim.

The above implies $\overline{\overline{p, i_{p}}}=\overline{\overline{s, i_{s}}}=\overline{\overline{t, j_{t}}}$, so either $s<p<t$ or $s<t \leq p$. If the latter holds, then since $\phi$ has left strict chain fibers, $i_{s} \geq j_{t} \geq i_{p}$ and also $i_{s} \leq i_{p}$ by isotonicity, and so $i_{s}=i_{p}=j_{t}$. Thus

$$
\overline{s, i_{s}} \leq \overline{t, j_{t}} \leq \overline{p, i_{p}}
$$

and since the extremes are equal, all three are equal contradicting the assumption that the two first are unequal.

Hence $s<p<t$. By assumption on the fibre of $\phi$ we have $i_{s} \geq i_{p}$ and by isotonicity $i_{s} \leq i_{p}$ and so $i_{s}=i_{p}$. Also by (16) and isotonicity

$$
i_{s} \geq j_{t} \geq j_{p} \geq j_{s} .
$$

By assumption $i_{s}=j_{s}$, and we get equalities everywhere and so $i_{p}=j_{p}$, as we wanted to prove.

By (16) we know that $i_{s} \geq j_{s}$. In case $i_{s}>j_{s}$ we have shown before Claim 4 that $m^{\ell}$ divides $m$. So suppose $i_{s}=j_{s}$. By the above two claims, the $x_{\overline{s, i_{s}}}$ in $m^{\ell}$ occurs only in $m_{i=j} \cdot m_{>s}^{i}$ and to a power less than or equal to that in $m_{i=j} \cdot m_{>s}^{j}$. Since $m^{j}$ divides $m x_{\overline{t, j_{t}}}$ and $\overline{s, i_{s}} \neq \overline{t, j_{t}}$ the power of $x_{\overline{s, i_{s}}}$ in $m^{j}$ is less than or equal to its power in $m$. 
Hence the power of $x_{s, i_{s}}$ in $m^{\ell}$ is less or equal to its power in $m$ and so by Claim $2 m^{\ell}$ divides $m$.

\section{REFERENCES}

1. Winfried Bruns and Udo Vetter, Determinantal rings, vol. 1327, Springer, 2006.

2. Alessio D'Alì, Gunnar Fløystad, and Amin Nematbakhsh, Resolutions of co-letterplace ideals and generalizations of Bier spheres, to appear in Transactions of the AMS, arXiv:1601.02793 (2016).

3. Viviana Ene, Syzygies of hibi rings, Acta Mathematica Vietnamica 40 (2015), no. 3, 403-446.

4. Viviana Ene, Jürgen Herzog, and Fatemeh Mohammadi, Monomial ideals and toric rings of Hibi type arising from a finite poset, European Journal of Combinatorics 32 (2011), no. 3, 404-421.

5. Valentin Féray and Victor Reiner, P-partitions revisited, Journal of commutative algebra 4 (2012), no. 1, 101-152.

6. Gunnar Fløystad, Bjørn Møller Greve, and Jürgen Herzog, Letterplace and co-letterplace ideals of posets, Journal of Pure and Applied Algebra 221 (2017), no. 5, 1218-1241.

7. Gunnar Fløystad and Amin Nematbakhsh, Rigid ideals by deforming quadratic letterplace ideals, to appear in Journal of Algebra, arXiv:1605.07417 (2016).

8. Christopher A. Francisco, Jeffrey Mermin, and Jay Schweig, Generalizing the Borel property, Journal of the London Mathematical Society 87 (2012), no. 3, 724-740.

9. Adriano M Garsia, Combinatorial methods in the theory of Cohen-Macaulay rings, Advances in Mathematics 38 (1980), no. 3, 229-266.

10. Jürgen Herzog and Dorin Popescu, Finite filtrations of modules and shellable multicomplexes, manuscripta mathematica 121 (2006), no. 3, 385-410.

11. Jürgen Herzog and Ngô Viêt Trung, Gröbner bases and multiplicity of determinantal and Pfaffian ideals, Advances in Mathematics 96 (1992), no. 1, 1-37.

12. Richard P Stanley, Ordered structures and partitions, vol. 119, American Mathematical Soc., 1972.

13. Richard P. Stanley, Enumerative combinatorics, Cambridge Studies in Advanced Mathematics, vol. 1, Cambridge University Press, 1997.

14. Bernd Sturmfels, Gröbner bases and Stanley decompositions of determinantal rings, Mathematische Zeitschrift 205 (1990), no. 1, 137-144.

Matematisk institutt, Universitetet i Bergen, Postboks 7803, 5020 Bergen

E-mail address: gunnar@mi.uib.no 